\title{
The Frog Motor Nerve Terminal Has Very Brief Action Potentials and Three Electrical Regions Predicted to Differentially Control Transmitter Release
}

\author{
Scott P. Ginebaugh, ${ }^{1}$ Eric D. Cyphers, ${ }^{1}$ Viswanath Lanka, ${ }^{1}$ Gloria Ortiz, ${ }^{2}$ Evan W. Miller,,${ }^{2,3}$ Rozita Laghaei, ${ }^{4}$ and \\ Stephen D. Meriney ${ }^{1}$ \\ ${ }^{1}$ Department of Neuroscience, Center for Neuroscience, University of Pittsburgh, Pittsburgh, Pennsylvania 15260, ${ }^{2}$ Departments of Chemistry, \\ ${ }^{3}$ Molecular and Cell Biology and the Helen Wills Neuroscience Institute, University of California, Berkeley, Berkeley, California 94720, and \\ ${ }^{4}$ Biomedical Application Group, Pittsburgh Supercomputing Center, Carnegie Mellon University, Pittsburgh, Pennsylvania 15213
}

\begin{abstract}
The action potential (AP) waveform controls the opening of voltage-gated calcium channels and contributes to the driving force for calcium ion flux that triggers neurotransmission at presynaptic nerve terminals. Although the frog neuromuscular junction (NMJ) has long been a model synapse for the study of neurotransmission, its presynaptic AP waveform has never been directly studied, and thus the AP waveform shape and propagation through this long presynaptic nerve terminal are unknown. Using a fast voltage-sensitive dye, we have imaged the AP waveform from the presynaptic terminal of male and female frog NMJs and shown that the AP is very brief in duration and actively propagated along the entire length of the terminal. Furthermore, based on measured AP waveforms at different regions along the length of the nerve terminal, we show that the terminal is divided into three distinct electrical regions: A beginning region immediately after the last node of Ranvier where the AP is broadest, a middle region with a relatively consistent AP duration, and an end region near the tip of nerve terminal branches where the AP is briefer. We hypothesize that these measured changes in the AP waveform along the length of the motor nerve terminal may explain the proximal-distal gradient in transmitter release previously reported at the frog NMJ.
\end{abstract}

Key words: Action potential; Nerve terminal; Neuromuscular junction; Synaptic transmission; Voltage imaging

Significance Statement

The AP waveform plays an essential role in determining the behavior of neurotransmission at the presynaptic terminal. Although the frog NMJ is a model synapse for the study of synaptic transmission, there are many unknowns centered around the shape and propagation of its presynaptic AP waveform. Here, we demonstrate that the presynaptic terminal of the frog NMJ has a very brief AP waveform and that the motor nerve terminal contains three distinct electrical regions. We propose that the changes in the AP waveform as it propagates along the terminal can explain the proximal-distal gradient in transmitter release seen in electrophysiological studies.

\section{Introduction}

Action potential (AP) invasion of the presynaptic nerve terminal activates voltage-gated calcium channels (VGCCs) positioned

\footnotetext{
Received Oct. 9, 2019; revised Mar. 2, 2020; accepted Mar. 9, 2020.

Author contributions S.P.G. and S.D.M. designed research; S.P.G., E.D.C., V.L., R.L., and S.D.M. performed research; G.O. and E.W.M. provided reagents and consultation for experiments; S.P.G. and S.D.M. analyzed data; S.P.G. and S.D.M. wrote the paper.

This work was supported by the University of Pittsburgh and NIH Grant NS090644 (S.D.M), by a Gilliam Fellowship from the Howard Hughes Medical Institute to G.0., and support from the NIH (R01NS098088) to E.W.M. We thank Steven B. Suway for writing the imaging delay routine for the Teensy USB development board.

The authors declare no competing financial interests.

Correspondence should be addressed to Stephen D. Meriney at meriney@pitt.edu.

https://doi.org/10.1523/JNEUROSCI.2415-19.2020

Copyright $(2020$ the authors
}

within active zones (AZs; highly organized sites of synaptic transmission that each contain an ordered array of synaptic vesicles and VGCCs) of the motor nerve terminal (Heuser and Reese, 1981; Pumplin et al., 1981; Robitaille et al., 1990; Cohen et al., 1991), resulting in an influx of calcium that triggers the exocytosis of neurotransmitter (Dodge and Rahamimoff, 1967; Meriney and Dittrich, 2013; Luo et al., 2015). Although the frog neuromuscular junction (NMJ) has been a model synapse for the study of synaptic transmission, its AP has never been directly studied due to the small diameter of the nerve terminal making traditional electrophysiological recording difficult.

The size and shape of the AP waveform greatly impacts transmitter release by controlling both the activation of VGCCs and the driving force for calcium entry through VGCCs while they 
are open (Sabatini and Regehr, 1997; Borst and Sakmann, 1999; Pattillo et al., 1999). The AP has been recorded from the soma of isolated adult frog spinal neurons (Dambach and Erulkar, 1973; Erulkar and Soller, 1980; Ovsepian and Vesselkin, 2006). However, the shape of the AP waveform at the adult motor nerve terminal is likely different because recordings in other neurons have found large discrepancies between AP waveforms at the soma and axon (Popovic et al., 2011; Hoppa et al., 2014; Rowan et al., 2016). Furthermore, the AP waveform has been shown to vary along the axons of other types of neurons due to several factors including the geometry of the axon and distributions of different types of voltage-gated ion channels (Hoppa et al., 2014; Rowan et al., 2016).

It was originally hypothesized that the AP is actively propagated along the length of the frog motor nerve terminals by voltage-gated sodium channels distributed along the entire length of the terminal (Katz and Miledi, 1965, 1968; Braun and Schmidt, 1966). However, the possibility that the AP may be passively propagated through these nerve terminal branches has also been proposed (Mallart, 1984; Robitaille and Tremblay, 1987). Furthermore, active propagation of the AP has been called into question by reports of proximal-distal gradients in neurotransmitter release magnitude along the length of frog motor nerve terminals (Mallart, 1984; Tremblay et al., 1984; D’Alonzo and Grinnell, 1985; Zefirov and Khalilov, 1985; Bennett et al., 1986a,b, 1989), and because these changes are not caused by differences in proximal-distal distribution of AZ structure (Pawson et al., 1998).

Here, we use a fast voltage-sensitive dye (Huang et al., 2015) to record the presynaptic AP waveform at the frog NMJ and investigate the changes to this waveform as it propagates along the motor nerve terminal. We show (1) that the AP waveform is very brief in duration, (2) the presynaptic terminal of the frog NMJ has three distinct electrical regions, and (3) that the changes in the AP waveform as it propagates along the length of the terminal not are caused by changes in nerve terminal geometry. Using imaged AP waveforms and previously validated computer simulations, we predicted effects of our recorded AP waveforms on presynaptic calcium entry and transmitter release, and hypothesize that changes in the shape of the AP may be responsible for proximal-distal differences in neurotransmitter release that were previously reported.

\section{Materials and Methods}

\section{Cutaneous pectoris nerve-muscle preparations}

Experiments were performed on cutaneous pectoris nerve-muscle preparations from both male and female adult frogs (Rana pipiens). Frogs were anesthetized in a $0.1 \%$ tricaine solution, then decapitated and double-pithed in adherence to the procedure approved by the University of Pittsburgh Institutional Animal Care and Use Committee. Preparations were dissected bilaterally and bathed in normal frog Ringer's solution [NFR; (in mu): $116 \mathrm{NaCl}, 2 \mathrm{KCl}, 5$ dextrose, $1 \mathrm{MgCl}_{2}, 1.8 \mathrm{CaCl}_{2}, \mathrm{pH}$ 7.3-7.4, and $10 \mathrm{~mm} \mathrm{BES].}$

\section{Voltage imaging}

The BeRST 1 dye (Huang et al., 2015) was stored in $5 \mathrm{~mm}$ aliquots in DMSO at $-80^{\circ} \mathrm{C}$. On the day of use, $0.5 \mu$ l of BeRST 1 dye stock solution was diluted into $4.5 \mu \mathrm{l}$ of DMSO initially, and then this mixture was diluted into $5 \mathrm{ml}$ of NFR (for a final BeRST 1 concentration of $0.5 \mu \mathrm{M}$ ). This final loading solution also contained $10 \mu \mathrm{g} / \mathrm{ml}$ of AlexaFluor 488conjugated $\alpha$-bungarotoxin (BTX) to counterstain postsynaptic receptors at the NMJ. To load nerve terminals with dye for the voltage-imaging procedure, the neuromuscular preparation was mounted over an elevated Sylgard platform in a $35 \mathrm{~mm}$ dish and incubated with the Berst 1 and BTX dye mixture for $90 \mathrm{~min}$. The dye-loaded and BTX-stained
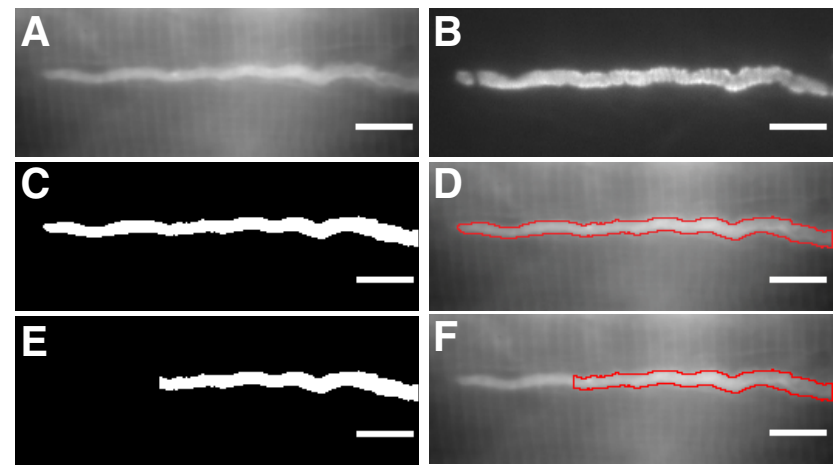

Figure 1. Imaging of the nerve terminal. $\boldsymbol{A}$, An image of a presynaptic motor nerve terminal stained with BeRST 1 dye. $\boldsymbol{B}$, The same nerve terminal as in $A$ stained with Alexa Fluor $488 \alpha$-BTX. C, An Otsu local thresholding algorithm applied to the terminal in $A$ to create an image mask. $\boldsymbol{D}$, An outline of the Otsu threshold image applied as an ROI for BeRST 1 dye imaging. $\boldsymbol{E}$, The 0 tsu local thresholding image with the end of the terminal removed for analysis. $\boldsymbol{F}$, The outline of the Otsu thresholding image with the end removed and applied as an ROI for the BeRST 1 dye imaging. Scale bars, $10 \mu \mathrm{m}$.

preparation was then rinsed with NFR, mounted on the microscope stage, and the nerve was drawn into a suction electrode for suprathreshold stimulation. If the BTX conjugated to AlexaFluor 488 did not completely block muscle contractions, $10 \mu \mathrm{M}$ curare was added to the imaging saline to completely block postsynaptic acetylcholine receptors and prevent nerve-evoked muscle contractions.

For voltage imaging, tissue was mounted on an Olympus BX61 microscope equipped with a $60 \times$ water-immersion objective. Nerve terminals were identified using the BTX staining, and this label was also used to bring the terminals into focus for voltage imaging. Superficial nerve terminals tended to load a higher concentration of BeRST 1 dye and produce noticeable less-noisy data than nerve terminals embedded deeper in the muscle. Thus, all terminals imaged were on the surface of the muscle. After locating a well-stained nerve terminal, we selected an imaging region-of-interest (ROI) of $\sim 80 \times 30 \mu \mathrm{m}$ that contained a large portion of the nerve terminal branch (Fig. $1 A, B$ ). All voltage imaging was performed in NFR at room temperature $\left(20-25^{\circ} \mathrm{C}\right)$.

Presynaptic nerve terminals in the frog cutaneous pectoris nervemuscle preparations were stimulated at $0.2 \mathrm{~Hz}$, and BeRST 1 dye fluorescence was recorded by an EMCCD camera (Pro-EM 512, Princeton Instruments). Image capture was coupled to illumination by a $640 \mathrm{~nm}$ laser (89 North laser diode illuminator) that only illuminated the tissue during the brief $100 \mu$ s image collection window. The entire AP waveform was sampled through a moving-bin acquisition scheme, where 100 bins were collected sequentially over the time course of an AP waveform that was elicited repeatedly at $0.2 \mathrm{~Hz}$. A custom routine on a Teensy 3.5 USB development board (PJRC) created a delay between the stimulation of the nerve and the triggering of the camera and laser. This delay was increased by $20 \mu$ s after each stimulation in the collection time course. After 100 bins were collected, the delay was reset to 0 . This process was repeated 5-20 times for each nerve terminal, creating 5-20 recordings of the full AP time course within the imaging ROI.

\section{Image and data analysis}

Images were analyzed in ImageJ and MATLAB (MathWorks) using custom written scripts. In ImageJ, the image stacks were stabilized for $x-y$ drift using an "align slices in stack" ImageJ plugin (https://sites-googlecom.pitt.idm.oclc.org/site/qingzongtseng/template-matching-ij-plugin; Tseng et al., 2011, 2012). Then, an Otsu local image threshold (Otsu, 1979) was applied to the average fluorescence $z$-projection of the stabilized BeRST 1 image stack to create an unbiased ROI selection containing the nerve terminal (Fig. 1C,D). The average signal inside a subsection of this ROI (depending on the experiment) was used as the nerve signal. For control experiments, a region at least $20 \mu \mathrm{m}$ from the end and last node of Ranvier (Fig. $1 E, F$ ) was used as the nerve signal (Fig. 2A). The region outside of the Otsu selected ROI (Fig. 1C,D) was 
Pre-Tetrodotoxin
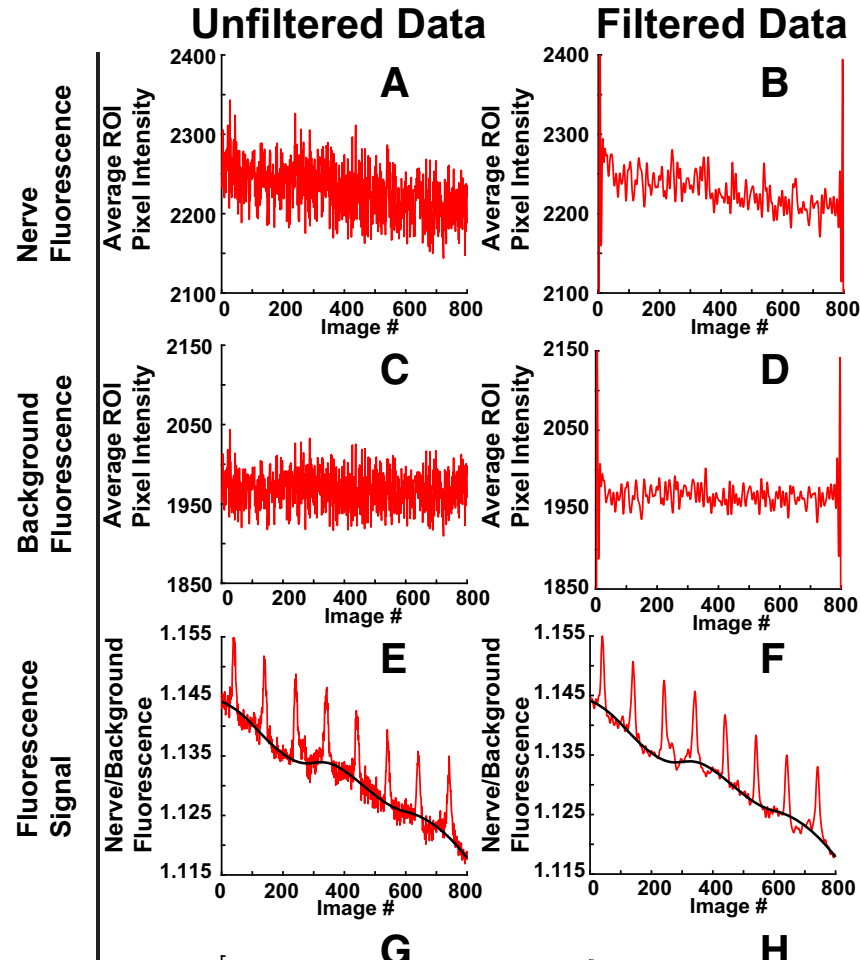

$\mathbf{F}$

$\mathrm{H}$

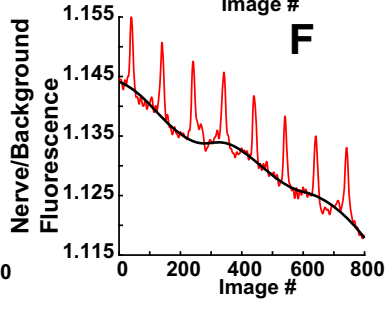

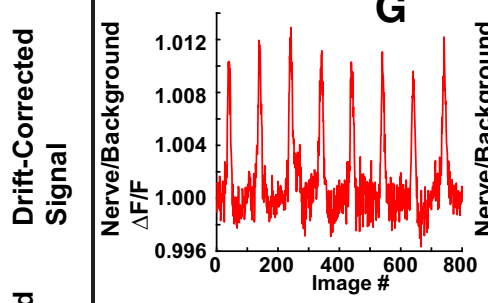
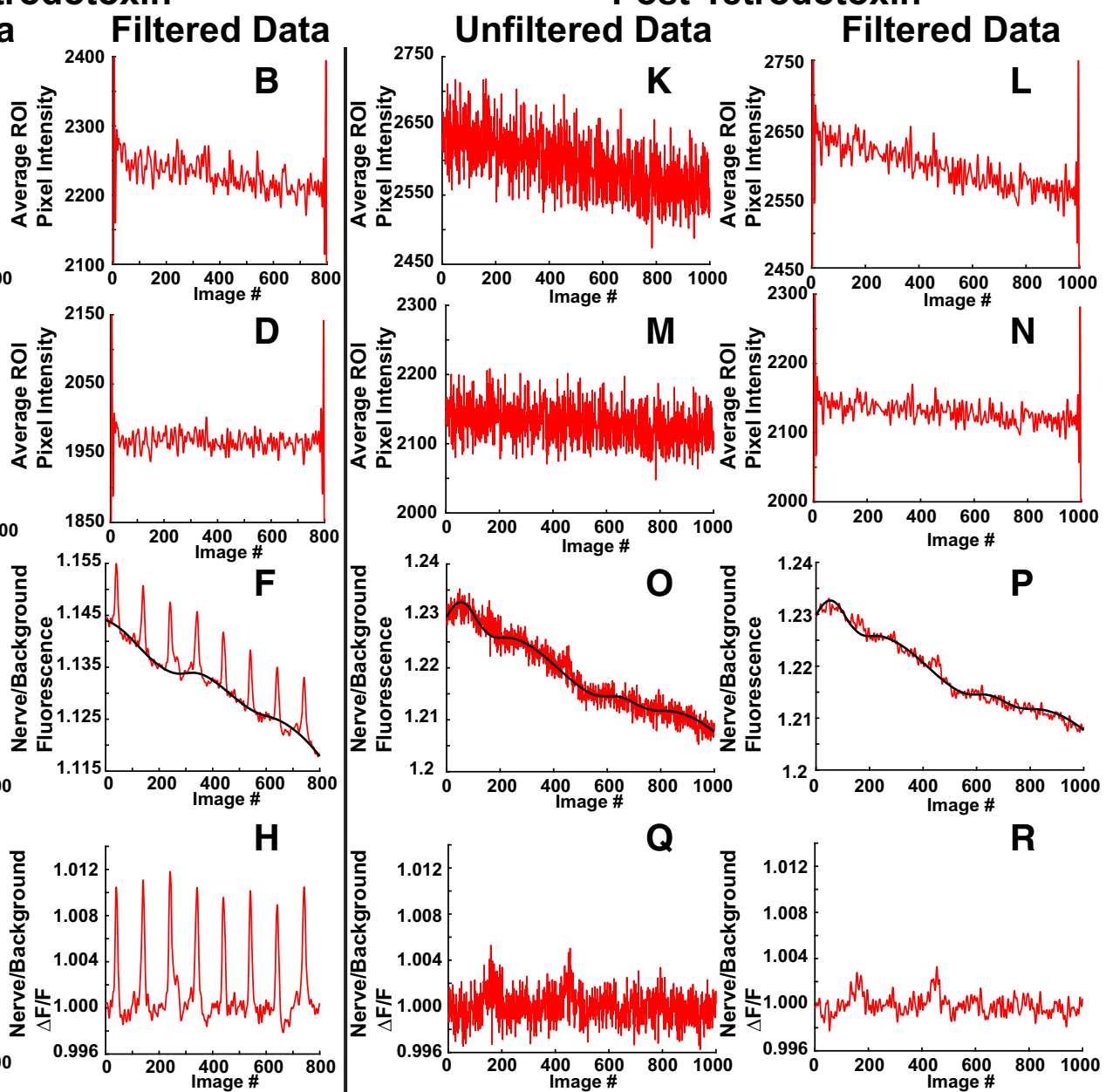

Post-Tetrodotoxin
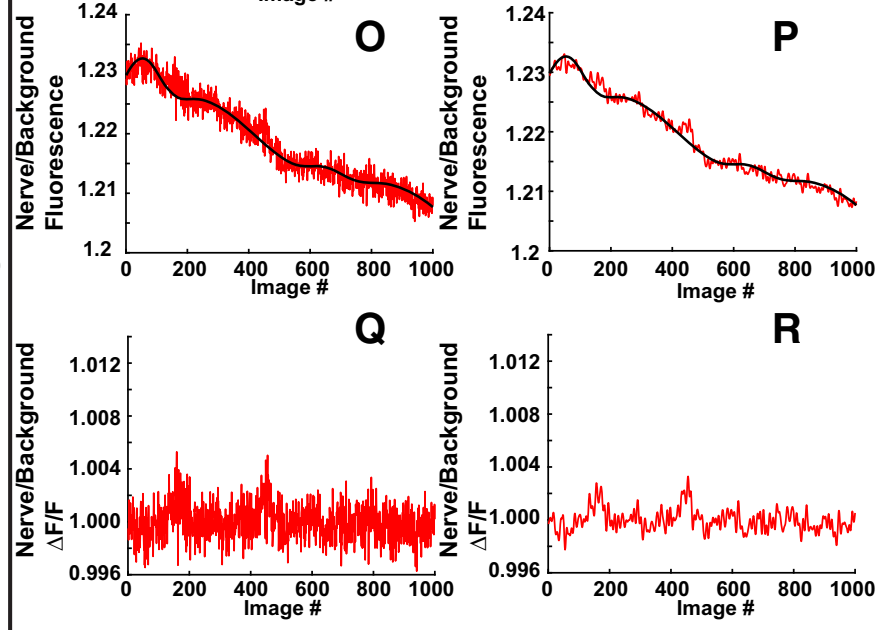

S

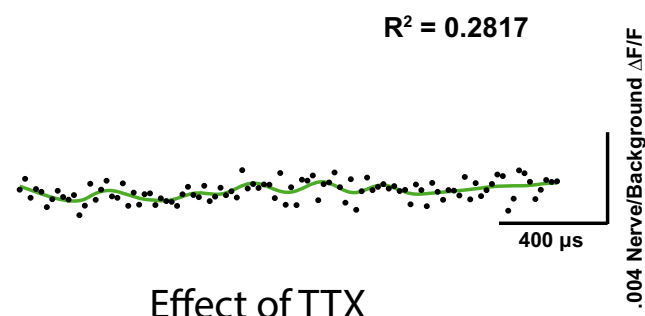

T

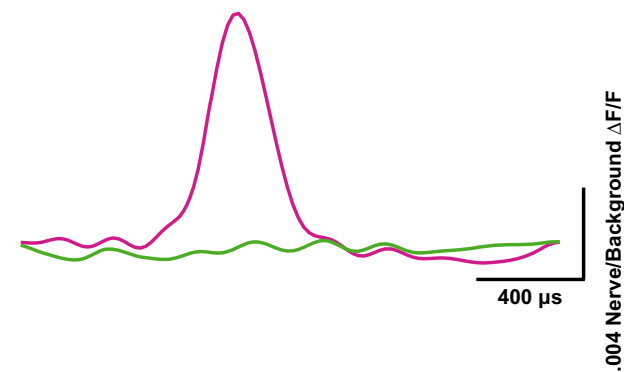

Figure 2. Analysis of the AP waveform and the impact of TTX. A, C, The unfiltered fuorescence signals from the nerve and background selection of 800 sequential images collected from a motor nerve terminal Rol. $\boldsymbol{B}, \boldsymbol{D}$, The nerve and background signals from $\boldsymbol{A}$ and $\mathbf{C l o w}$-pass filtered at $4 \mathrm{kHz}$, including the ringing artifact on the edges of the recording (truncated in the vertical axis). $\boldsymbol{E}, \boldsymbol{F}$, The unfiltered and filtered fluorescence signals, created by dividing the fluorescence values in $\boldsymbol{C}$ from $\boldsymbol{A}$, or $\boldsymbol{D}$ from $\boldsymbol{B}$, respectively, showing eight AP waveforms (red) with a cubic spline fit (black) thorough the unstimulated (baseline) points. $\boldsymbol{G}, \boldsymbol{H}$, The unfiltered and filtered fluorescence signal after correcting for the baseline by dividing the cubic spline from the fluorescence signal in $\boldsymbol{E}$ and $\boldsymbol{F}$. $\boldsymbol{I}$, The average of the 8 unfiltered AP waveforms from $\boldsymbol{G}$ (black dots) compared with the average of the eight filtered AP waveforms from $\boldsymbol{H}$ (magenta line). Comparing the two provides an $R^{2}$ value, which represents a measure of the fit weighted by the signal strength compared with the baseline noise. J, The average filtered AP waveform from $I$, fit with an interpolating cubic spline and then normalized to the baseline of the trace (based on the average value of the first 15 points of the average filtered AP waveform). $\boldsymbol{K}-\boldsymbol{N}$, The unfiltered and filtered fluorescence signals from the nerve and background selection of 1000 images of the same nerve terminal as in $\boldsymbol{A}-\boldsymbol{D}$ but in the presence of $1 \mu \mathrm{m}$ TTX. $\mathbf{O}, \boldsymbol{P}$, The unfiltered and filtered 
averaged and used as the background signal (Fig. 2C). Both the background and nerve signals were then low-pass filtered off-line $\left(f_{\text {pass }}=4\right.$ $\mathrm{kHz}$; Fig. $2 B, D$ ). We processed our signals both with and without filtering. We divided the unfiltered and filtered background fluorescence (Fig. 2C,D) from the unfiltered and filtered nerve fluorescence (Fig. $2 \mathrm{~A}, B)$, respectively, to give us the unfiltered and filtered fluorescence signal (Fig. 2E,F). To correct for $z$-axis drift (during which the live image could drift slightly in a nonlinear fashion; Fig. $2 E, F$ ), we fit a cubic B-spline to the points in the fluorescence time course during which there was no AP stimulation (the first and last 15 points of the 100 total points in each series). We then divided this spline from each point in the fluorescence signal to give us a $\Delta F / F$ fluorescence signal that did not fluctuate as a result of drift of the nerve-muscle preparation (Fig. 2G,H).

The APs from the $\Delta \mathrm{F} / \mathrm{F}$ fluorescence signal were then averaged to create a single AP waveform (Fig. 2I). To prevent discrepancies caused by including excessively noisy data, this analysis was performed both with (Fig. 2B,D,F,H) and without (Fig. 2A,C,E,G) the low-pass filter. We then calculated the $R^{2}$ value between the averaged unfiltered and filtered AP waveforms (Fig. 2I). Because we are not using a linear model, $R^{2}$ is not an exact measure of fit between the filtered and unfiltered data, and is greatly affected by the ratio of the signal strength to the baseline noise. This provides a benefit in that not only will the $R^{2}$ value detect a poor fit between the filtered and unfiltered data, but will also provide a low value if the AP signal is not sufficiently detected compared with the noise (Fig. $2 S$ ). This is essential because normalizing any small signal on an otherwise flat waveform could appear as an AP. Thus, in this instance the $R^{2}$ is analogous to the range-normalized root-mean-square deviation. However, $R^{2}$ is less sensitive to outliers than the range-normalized rootmean-square deviation and provides easier-to-interpret results. Thus, we used the $R^{2}$ value only as a heuristic method to determine the quality of our recordings, and not for any statistical purposes.

For some recordings, image artifacts in the background (for example, a free-floating piece of connective tissue stained with BeRST 1 dye) resulted in noisy data due to the improper background use for calculating the nerve signal. If the $R^{2}$ was $<0.95$ for the full image (Fig. 6) and $20 \mu \mathrm{m}$ ROI data (Fig. 7) or 0.85 for the 10-pixel diameter small-circle ROI data (Fig. 8), a subsection of the background near the nerve terminal of $\sim 15 \times 30 \mu \mathrm{m}$ was used rather the complete region outside the Otsu-selected ROI. If neither the full background selection nor the smaller background subsection resulted in an $R^{2}$ value higher than the values listed above, the recording was considered too noisy and was not included in the data analysis. If the recording was of high enough quality for analysis, the average AP was normalized to the baseline of the trace (the average value of the first 15 points), then cubic spline interpolation at an oversampled time resolution of $2 \mu$ s was fit to the filtered data, and the full-width at half-maximum (FWHM) of the AP waveform was calculated (Fig. 2J).

We performed this procedure on the same nerve terminal both in the presence and absence of $1 \mu \mathrm{m}$ tetrodotoxin (TTX), and showed that these optically recorded AP signals were sodium channel dependent based on their elimination in the presence of tetrodotoxin (Fig. $2 \mathrm{~K}-\mathrm{T}$ ).

Because the BeRST 1 dye stains all lipid membranes and connective tissues after bath application, the background was proportional to the total fluorescence, rather than being limited to standard camera shot noise. Thus, the background was divided, rather than subtracted, from $\leftarrow$

fluorescence signals, created by dividing the fluorescence values in $\boldsymbol{M}$ from $\boldsymbol{K}$ or $\boldsymbol{N}$ from $\boldsymbol{L}$, respectively, showing the signal (red) with a cubic spline fit (black) thorough the unstimulated (baseline) points. $\boldsymbol{Q}, \boldsymbol{R}$, The unfiltered and filtered fluorescence signal after correcting for the baseline by dividing the cubic spline from the fluorescence signal in $\mathbf{O}$ and $\boldsymbol{P}$. S, The average of the 10 stimulation cycles of the unfiltered signal from $\mathbf{Q}$ (black dots) compared with the average of the 10 stimulation cycles of the filtered signal filtered from $\boldsymbol{R}$ (green line). The low $R^{2}$ value shows that the TTX eliminates the presence of the sodium-dependent AP waveform. $T$, A comparison of the average filtered fluorescent signal before (magenta) and after (green) the application of TTX. the nerve signal to give us the fluorescence value used for analysis. Dividing the background also provided an additional advantage of eliminating the ringing artifacts caused by the low-pass filter overshooting the time domain. Furthermore, even when the ringing artifacts were removed, dividing the background still resulted in a less noisy signal (determined by higher $R^{2}$ values) than subtraction (Fig. 3 ).

Based on previous research, we expected only a minor distortion caused by our use of $100 \mu$ s collection windows (Popovic et al., 2011). To confirm this prediction, we first created a theoretical AP waveform in MATLAB using a normalized Gaussian with a standard deviation of 100 $\mu$ s that consisted of 20,000 points over 2000 time units, creating a waveform with a FWHM of $235 \mu$ s (10 points per $\mu$ s across a $2000 \mu$ s window created a smooth curve). We sampled this waveform every $20 \mu \mathrm{s}$ with a $100 \mu$ s recording window (averaging all points within the window), mimicking our imaging procedure, or every $20 \mu$ s with a $50 \mu$ s recording window, and compared the results to the true waveform we created. We found that using a moving-bin sampling approach with a 50 $\mu$ s or $100 \mu$ s recording window created only a small increase in the FWHM of the theoretical AP waveform (1.5 or 4.2\%, respectively; Fig. $4 A)$. We then confirmed that our sampling scheme had little effect on our experimental data by recording from a single motor nerve terminal with our $20 \mu$ s moving-bin acquisition scheme using 20, 50, and $100 \mu$ s recording windows, and found little difference in the predicted waveforms (Fig. 4B). However, the data collected with the $100 \mu$ s recording window was less noisy (and had better $R^{2}$ values) than the data recorded with the 50 and $20 \mu$ s recording windows for the same number of images (Fig. $4 B$ ). Thus, we chose to use $100 \mu$ s recording windows and we chose not to correct for the small distortion in the AP waveform.

We made several attempts to calibrate the voltage by zeroing membrane potential using gramicidin (Podleski and Changeux, 1969; Meunier, 1984; Maric et al., 1998; Hoppa et al., 2014). However, we found that we were unable to block tissue movement caused by gramicidin even in the presence of a myosin chain blocker (Heredia et al., 2016). Thus, we chose to only report changes in the width of the voltageimaging-measured AP waveforms, as is common in other papers with voltage imaging (Popovic et al., 2011; Ford and Davis, 2014; Rowan et al., 2014, 2016; Rowan and Christie, 2017). As such, the focus of this report is on the duration of presynaptic AP waveforms.

\section{MCell simulations}

MCell (https://mcell.org) was used to computationally study the impact of the experimentally recorded AP waveforms on AP-triggered calcium entry, and the impact of these waveforms on vesicle release from a modeled presynaptic nerve terminal AZ based on the frog NMJ. In brief, MCell is a particle-based, stochastic diffusion-reaction simulator that tracks the diffusion of particles through arbitrarily complex 3D structures (Kerr et al., 2008).

Here, we used two different models. First, to measure the impact of the AP waveforms on VGCCs, a model that consisted of a box containing 10,000 VGCCs was created. This box model simply allowed for faster simulations and easier analysis of these VGCC characteristics because these simulations did not need to keep track of numerous other parameters. During a MCell run, an AP waveform triggered VGCCs to open stochastically according to an AP driven Markov-model ion channel gating scheme (Fig. 5). When open, calcium ions entered the simulation environment from VGCCs at a rate determined by the instantaneous driving force. The total calcium inside the box and the probability of VGCCs opening were recorded during the AP waveform input.

Second, we used a previously-described frog computational model of a frog motor nerve terminal active zone to measure the impact of the experimental AP waveforms on transmitter release, as has been described previously (Dittrich et al., 2013; Ma et al., 2014; Homan et al., 2018; Laghaei et al., 2018). This single AZ model contained 26 synaptic vesicles and 26 VGCCs [driven by the same voltage-dependent gating scheme (Fig. 5) as in the box model] arranged in a double row at a 1:1 VGCC to synaptic vesicle stoichiometry (Luo et al., 2011). The bottom of each vesicle contained synaptotagmin-1 and second sensor binding sites (similar to synaptotagmin-7) responsible for triggering vesicle release (Ma et al., 2014). During an AP waveform, calcium diffused into the AZ model and 

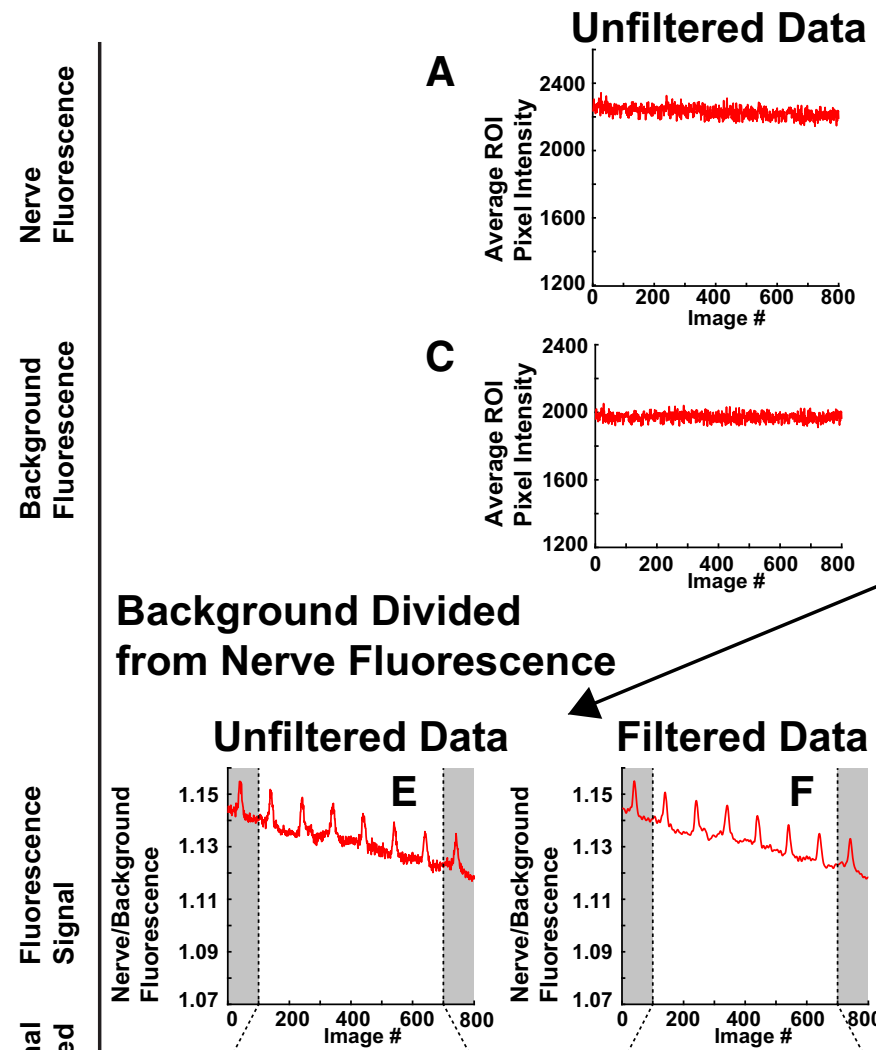

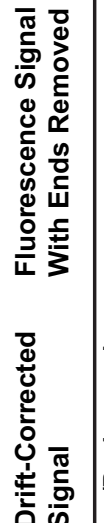
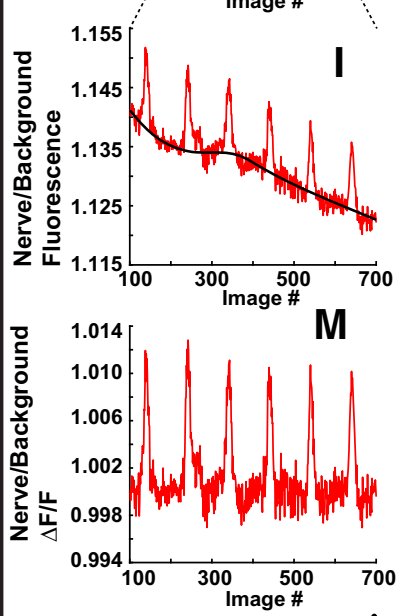

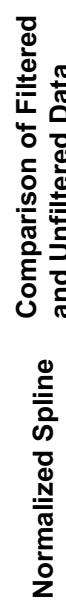
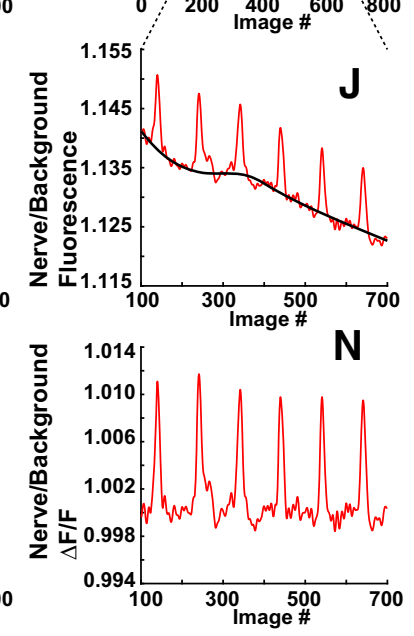

Filtered Data

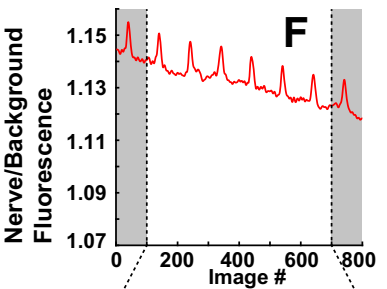

Q

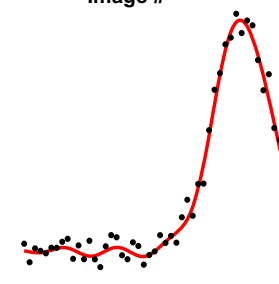

$R^{2}=0.9809$

S

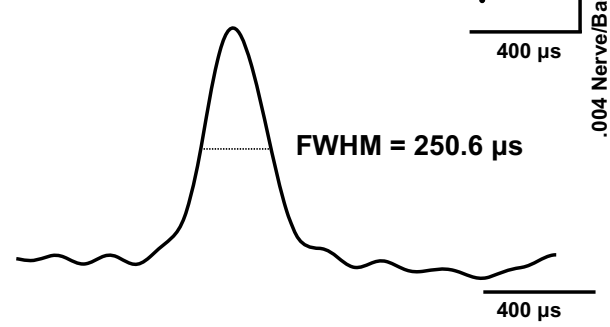

Filtered Data

B

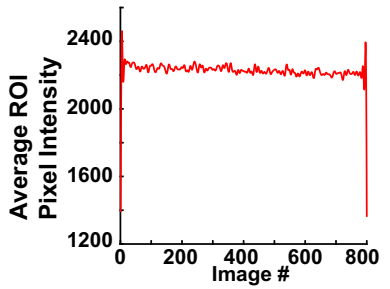

D
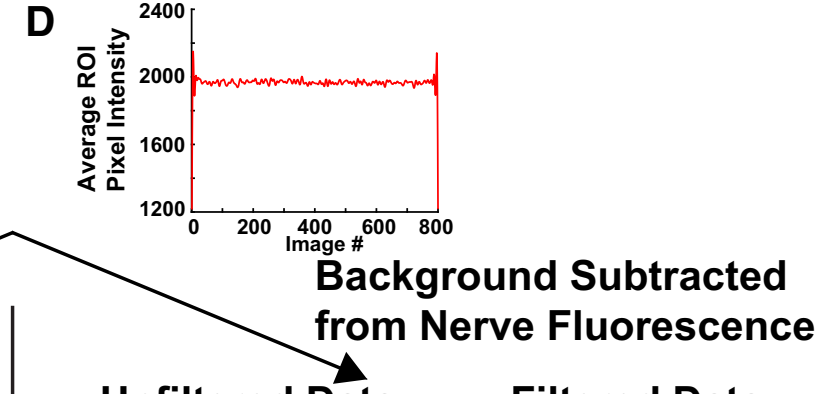

Filtered Data
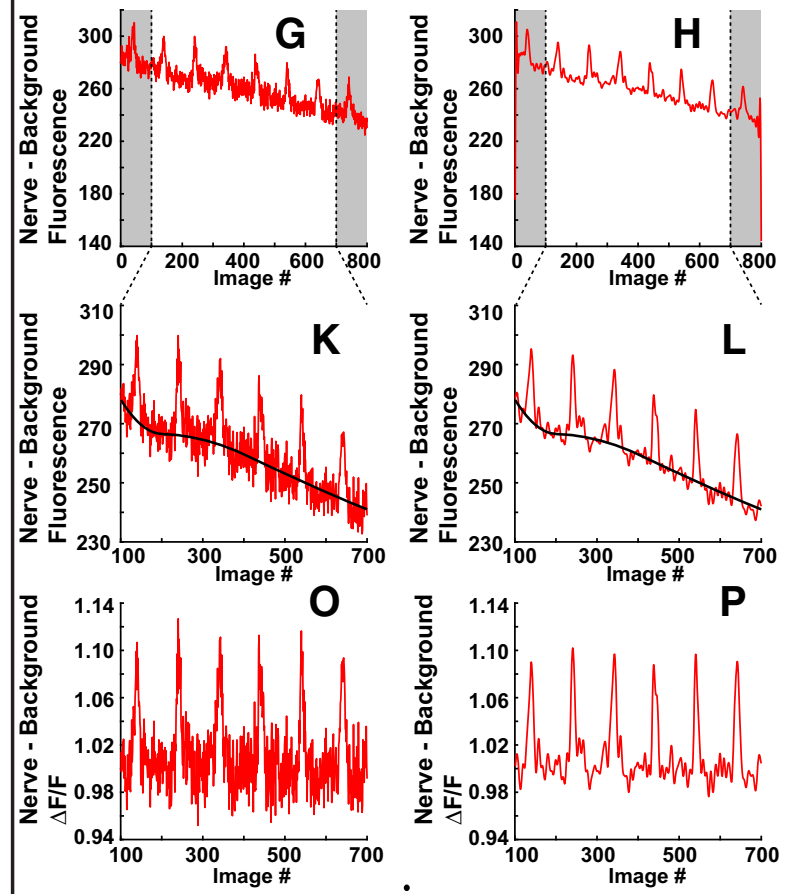

R
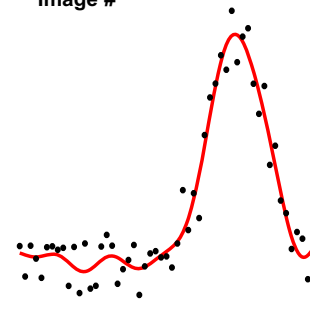

$$
R^{2}=0.9227
$$

$\mathbf{T}$

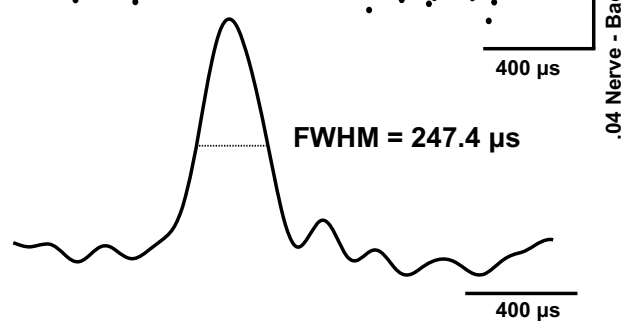

Figure 3. Dividing the background signal from the nerve signal produces a better signal-to-noise waveform than subtracting the background signal. $\boldsymbol{A}-\boldsymbol{D}$, The unfiltered and filtered data from the same 800 images of a nerve terminal shown in Figure $2 A-D$. Here, the $y$-axis is expanded to show the full ringing artifact in the filtered data. $E, F$, The unfiltered and filtered fluorescence signals created by dividing the background fluorescence from the nerve fluorescence. $\boldsymbol{G}, \boldsymbol{H}$, The unfiltered and filtered fluorescence signals created by subtracting the background 


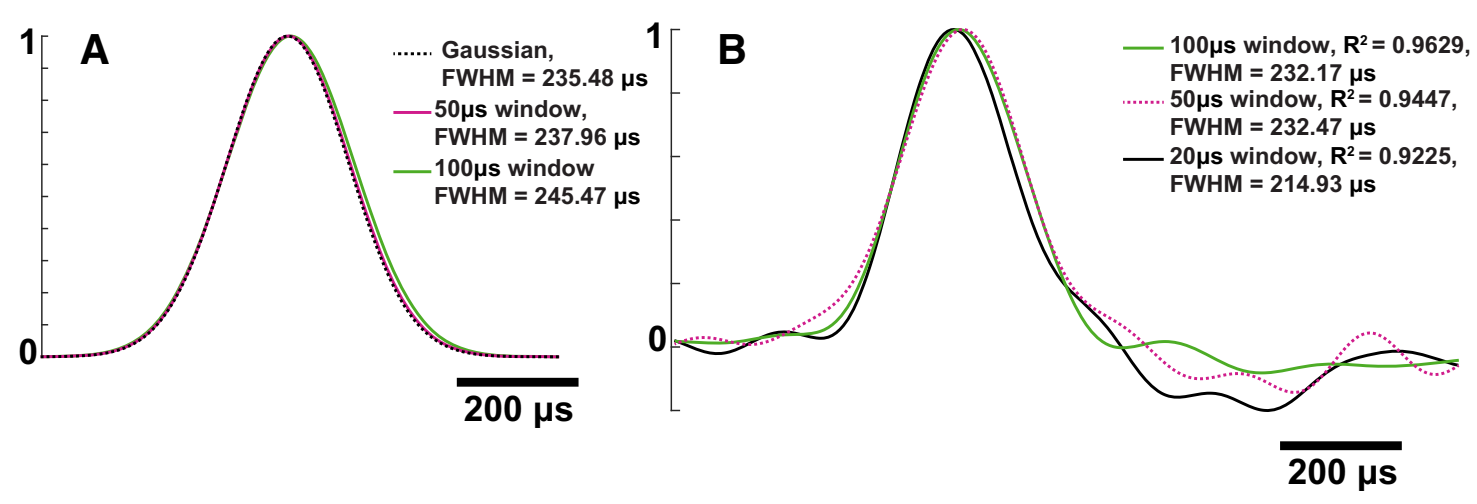

Figure 4. A $100 \mu$ s recording window only causes a small distortion of the AP waveform, but improves the recording quality (as judged by the $R^{2}$ values). $A, A$ theoretical waveform created from a normalized Gaussian curve (black dots), compared with a waveform created by sampling the Gaussian with a $50 \mu$ s recording windows every $20 \mu s$ (magenta) and a waveform created by sampling the Gaussian with a $100 \mu$ s recording windows every $20 \mu$ s, mimicking our imaging procedure (green). This creates a theoretical 1.5 and $4.2 \%$ increase in the AP FWHM for the 50 and $100 \mu$ s recording windows, respectively. B, Normalized AP waveforms created from 1000 images each, with $20 \mu \mathrm{s}$ (black), $50 \mu \mathrm{s}$ (magenta), or $100 \mu \mathrm{s}$ (green) moving bin recording windows. The AP FWHM from the $100 \mu$ s recording window is slightly larger than from the $20 \mu$ s recording window, but has a better $R^{2}$ value for the same number of images.

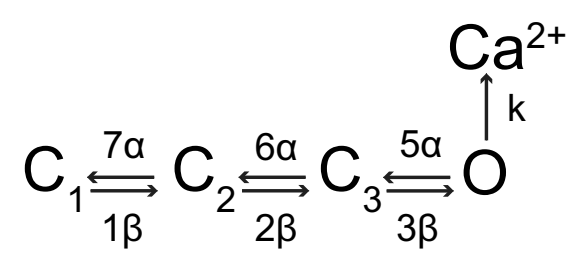

Figure 5. The four-state Markov chain VGCC gating scheme that determines the opening behavior of VGCCs in both the frog AZ MCell model and the 10,000 VGCC MCell box simulation.

bound to either calcium buffer (which was distributed evenly throughout the terminal) or to the calcium sensor sites (synaptotagmin-1 or second sensor sites) on the synaptic vesicles. When a sufficient amount of calcium was bound to the sensor sites (Dittrich et al., 2013; Ma et al., 2014), the vesicle was considered to be "released". Because these simulations are stochastic, 4800 simulations were run for each waveform input.

The only differences between the previous reported frog AZ model and the AZ model used in this work were different AP waveform inputs to the model (Figs. $7 F, 12 A$ ), and new rate constants for the AP driven Markov chain ion channel gating scheme which drives the behavior of VGCCs in our model (Fig. 5; Dittrich et al., 2013). This gating scheme, like the scheme used in previous models, is a linear four-state model with three closed states and one open state. When in the open state, calcium ions enter the simulations environment from VGCCs with Poisson probability based on the parameter $k=(-\gamma \mathrm{G} / 2 \mathrm{e}) \times\left(\mathrm{V}_{\mathrm{m}}-\mathrm{E}_{\mathrm{Ca}}\right)$, where $V_{\mathrm{m}}$ is the membrane voltage (determined by the AP waveform input), $G=2.4$ is the channel conductance, $E_{\mathrm{Ca}}=+50 \mathrm{mV}$ is the equilibrium potential, $e$ is the elementary charge, and $\gamma=0.9$ is a scaling constant to account for the fact that the

$\leftarrow$

fluorescence from the nerve fluorescence. Notice how subtraction does not remove the ringing artifact in the filtered data. $\boldsymbol{I}-\boldsymbol{L}$, The fluorescence signals (red lines) with the outer 100 data points on each side $(\boldsymbol{E}-\boldsymbol{H}$, gray bars) removed to exclude the ringing artifact remaining in the filtered fluorescence data created by subtraction $(\boldsymbol{H})$, fit with a cubic spline through the unstimulated points (black line). $\boldsymbol{M}-\boldsymbol{P}$, The unfiltered and filtered fluorescence signal after correcting for the baseline by dividing the cubic spline from the fluorescence signals. $\mathbf{Q}$, $\boldsymbol{R}$, The average of 6 unfiltered AP waveforms (black dots) compared with the average of six filtered AP waveforms (red line). Notice how subtracting the background created a noisier signal and weaker signal-to-noise ratio, and therefore a lower $R^{2}$ value, even with the removal of the ringing artifact. $S, T$, The average filtered AP waveforms, fit with interpolating cubic splines and then normalized to the baseline of the traces (based on the average value of the first 15 points of the average filtered AP waveforms). channel conductance was originally determined in $2 \mathrm{~mm}$ extracellular calcium. The rates for forward transitions between the states were determined by rate constants multiplied by the voltage-dependent parameter $\alpha$, and reversal rates were determined by rate constants multiplied by the voltage-dependent parameter $\beta$, where $\alpha=0.06 \exp \left(\left(V_{\mathrm{m}}+24\right) / 14.5\right)$ and $\beta=1.7 /\left(\exp \left(\left(V_{\mathrm{m}}+34\right) / 16.9\right)+1\right)$. These rate constants were parametrized based on an experimentally measured whole-cell calcium current (Pattillo et al., 2001; Dittrich et al., 2013). $\alpha$ and $\beta$ were the same as used in previous models, but the forward and reversal rate constants were reparametrized to fit the predicted calcium current activated by our newly recorded AP waveform.

To modify our experimentally imaged APs for input into MCell (importantly, to set the AP waveform to a membrane voltage, and to put the waveform in a MCell-friendly format), we normalized the waveforms to a resting potential of $-60 \mathrm{mV}$ and a peak voltage of $30 \mathrm{mV}$. To remove fluctuations near the onset of the rising phase of the APs caused by noise in the experimental data, we standardized the beginning of the rising edge of each AP by replacing the first $10 \%$ of the rising edge with a line that had the same slope as the next $10 \%$ of the rising edge. To reduce fluctuations caused by noise after repolarization of the APs, we smoothed the afterhyperpolarization phase of each AP with a cubic polynomial (compare Figs. $7 F, 12 A)$.

\section{NEURON simulations}

A passive model of a frog motoneuron nerve terminal was simulated in NEURON, version 7.7 (https://www.neuron.yale.edu/neuron/; Hines and Carnevale, 1997, 2001). This model was a single tube with a length of $200 \mu \mathrm{m}$ (approximately the average length of our recorded nerve terminal branches) and a diameter of $3.14 \mu \mathrm{m}$ (the average diameter of our recorded nerve terminals). The axial resistance was set to $110 \Omega \mathrm{cm}$ (Miralles and Solsona, 1996), the leak conductance was set to $1 \mathrm{e}-5 \mathrm{~S} / \mathrm{cm}^{2}$ (Lindgren and Moore, 1989), the membrane capacitance was set to 1 $\mu \mathrm{F} / \mathrm{cm}^{2}$, and the temperature was set to $20^{\circ} \mathrm{C}$. A recorded AP waveform was input into one end of the passive tube with a single electrode voltage clamp, and the shape and FWHM of the AP waveform was recorded as it propagated passively along this modeled long nerve terminal branch.

\section{Experimental design and statistical analysis}

GraphPad Prism (GraphPad Software), MATLAB (MathWorks), SPSS (IBM), and Excel (Microsoft) were used for analysis. All values are reported as mean \pm SEM (except where noted). The $\alpha$ for statistical significance was set to $p<0.05$. To determine normality, D'Agostino and Pearson test and Shapiro-Wilks test were used. In general, the FWHM data were not normally distributed (Fig. 6). Thus, all statistical tests were nonparametric. For paired datasets, the Wilcoxon matched-pairs signed rank test was used. For comparisons of multiple paired groups, the Friedman test was used and significance between groups was determined with Dunn's multiple post hoc comparisons test. For correlation tests, 


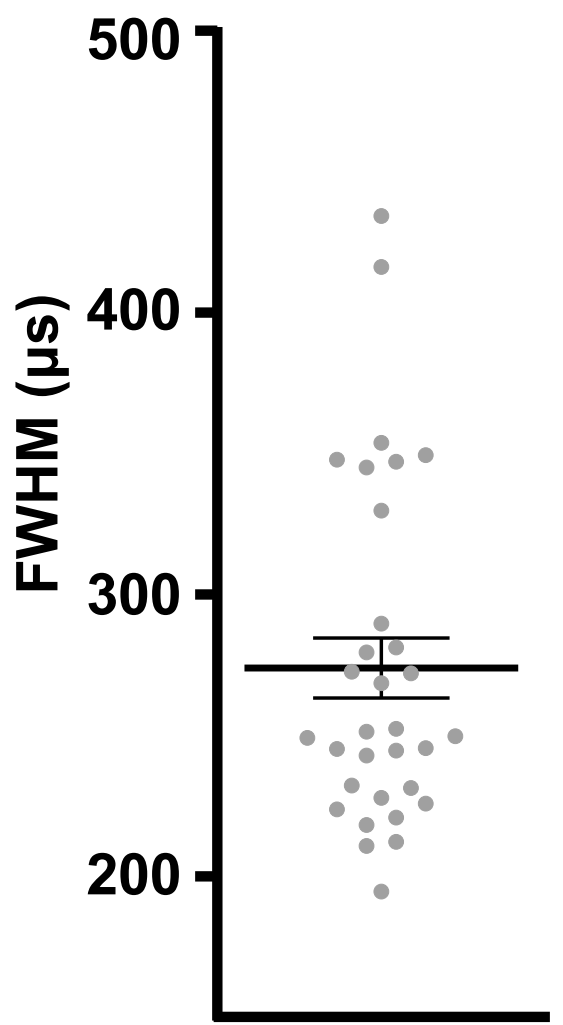

Figure 6. A scatter plot showing the range of AP durations (FWHM) from all control images (line $=$ mean \pm SEM), where the nerve ROI selection was at least $20 \mu \mathrm{m}$ from both the end of the terminal and the last node of Ranvier. The AP waveform in the frog motor nerve terminal is very brief and can vary between terminals.

Kendall's tau coefficient test with Bonferroni's correction was used. In all figures, ${ }^{*} p<0.05,{ }^{* *} p<0.01,{ }^{* * *} p<0.001,{ }^{* * *} p<0.0001$. A priori power tests were not performed, but the number of synapses imaged and animals used are similar to those generally used in the field.

\section{Results}

APs at the adult frog NMJ are brief in duration

To date, there have been several studies of the AP waveforms at small nerve terminals using voltage-sensitive dyes (Popovic et al., 2011; Rowan et al., 2014, 2016). However, there have been no AP waveform measurements at the long, linear, adult motor nerve terminals present at the frog NMJ. Using a live-cell imaging approach, we measured the response of a fast voltage-sensitive dye (BeRST 1) to AP waveforms propagating along the frog motor nerve terminal (although the temporal resolution of BeRST 1 has not been recorded, dyes with a similar chemical structure respond to changes in membrane potential in $\sim 25$ ns; Beier et al., 2019). For all experiments, we measured the FWHM to characterize the duration of the AP.

We found the AP in the adult frog motor nerve terminal to be very brief [the average FWHM of all control recordings $(n=32)$ was $273.9 \pm 10.7 \mu$ s; Fig. 6]. This is much briefer than might have been expected based on AP waveforms previously recorded from isolated adult frog spinal neurons (Dambach and Erulkar, 1973; Erulkar and Soller, 1980; Ovsepian and Vesselkin, 2006) and cultured embryonic frog motoneuron varicosities (Yazejian et al., 1997; Pattillo et al., 2001), where AP FWHM values range between 800 and $1200 \mu$ s. Interestingly, we also found a relatively large variability in the measured AP FWHM values across different NMJs (ranging from $\sim 200$ to $\sim 400 \mu$ sWHM).
This is consistent with the large range in quantal content observed across individual NMJs (Laghaei et al., 2018), even when corrected for nerve terminal length (Grinnell and Herrera, 1980). Therefore, the AP duration could be one factor that contributes to this variability.

\section{Differences in AP duration along the length of motor nerve} terminal branches reveal three distinct electrical regions Previous studies have shown that transmitter release varies along the length of the frog NMJ (Mallart, 1984; Tremblay et al., 1984; D'Alonzo and Grinnell, 1985; Zefirov and Khalilov, 1985; Bennett et al., 1986a,b, 1989). There is some debate as to the average magnitude and distribution of these proximal-distal changes: some studies suggest a continuous proximal-distal decrease in transmitter release (Bennett et al., 1986a,b, 1989), whereas others suggest that the transmitter release is greatest near the point at which the axon enters the presynaptic nerve terminal near the last node of Ranvier, reduces slightly but maintains a consistent level throughout the middle $60-90 \%$ of the nerve terminal, and then is reduced near the ends of the nerve terminal (D'Alonzo and Grinnell, 1985). Initially, this behavior was hypothesized to have been caused by proximal-distal changes in the structure of the AZs. However, freeze-fracture electron microscopy studies subsequently showed that there are no proximal-distal changes in AZ structure (Pawson et al., 1998). Thus, we tested the hypothesis that these proximal-distal changes in transmitter release could be caused by changes in the AP waveform as it propagates through the nerve terminal.

To study the AP waveform along the length of frog motor nerve terminal branches, we performed our voltage-imaging procedure near the entry of the axon into the presynaptic nerve terminal just after the last node of Ranvier and again at the end of a nerve terminal branch (Fig. $7 A, B$ ). In addition to the BeRST 1 images, we also documented the full length of NMJ nerve terminal branches we were studying using Alexa Fluor 488 BTX images, which were combined using image stitching (Preibisch et al., 2009) to create images of the full length of the nerve terminal branches under study. We then measured the length of the recorded nerve terminal branches using a skeletonizing method (Arganda-Carreras et al., 2010; Fig. 7C). Because the average nerve terminal branch length was $\sim 200 \mu \mathrm{m}(194.6 \pm 7.8 \mu \mathrm{m})$, we analyzed the voltage-imaging data using multiple equally-distributed ROIs, each $20 \mu \mathrm{m}$ in length, each corresponding to $\sim 10 \%$ of the nerve terminal branch length. In particular, we focused on the first, second, and third $20 \mu \mathrm{m}$ ROIs starting from the last node of Ranvier compared with the first, second, and third $20 \mu \mathrm{m}$ ROIs starting from the end of the nerve terminal (Fig. 7A,B). This analysis revealed significant differences in the AP waveform duration near the last node of Ranvier and near the end of the nerve terminal, but no significant differences along the extended middle portion of these long nerve terminal branches (Fig. 7D-F).

These data do not suggest that the AP waveform duration decreases linearly with distance along the presynaptic nerve terminal branch, but only changes immediately after the axonal entry into the nerve terminal and near the tip of the nerve terminal branch. Based on these data, we propose that there are three distinct regions in the presynaptic nerve terminal of the frog NMJ where electrical signals are different: a beginning region immediately after the last node of Ranvier, a middle region consisting of $60-80 \%$ of the nerve terminal with a relatively consistent AP duration, and an end region near the tip of nerve terminal branches. 

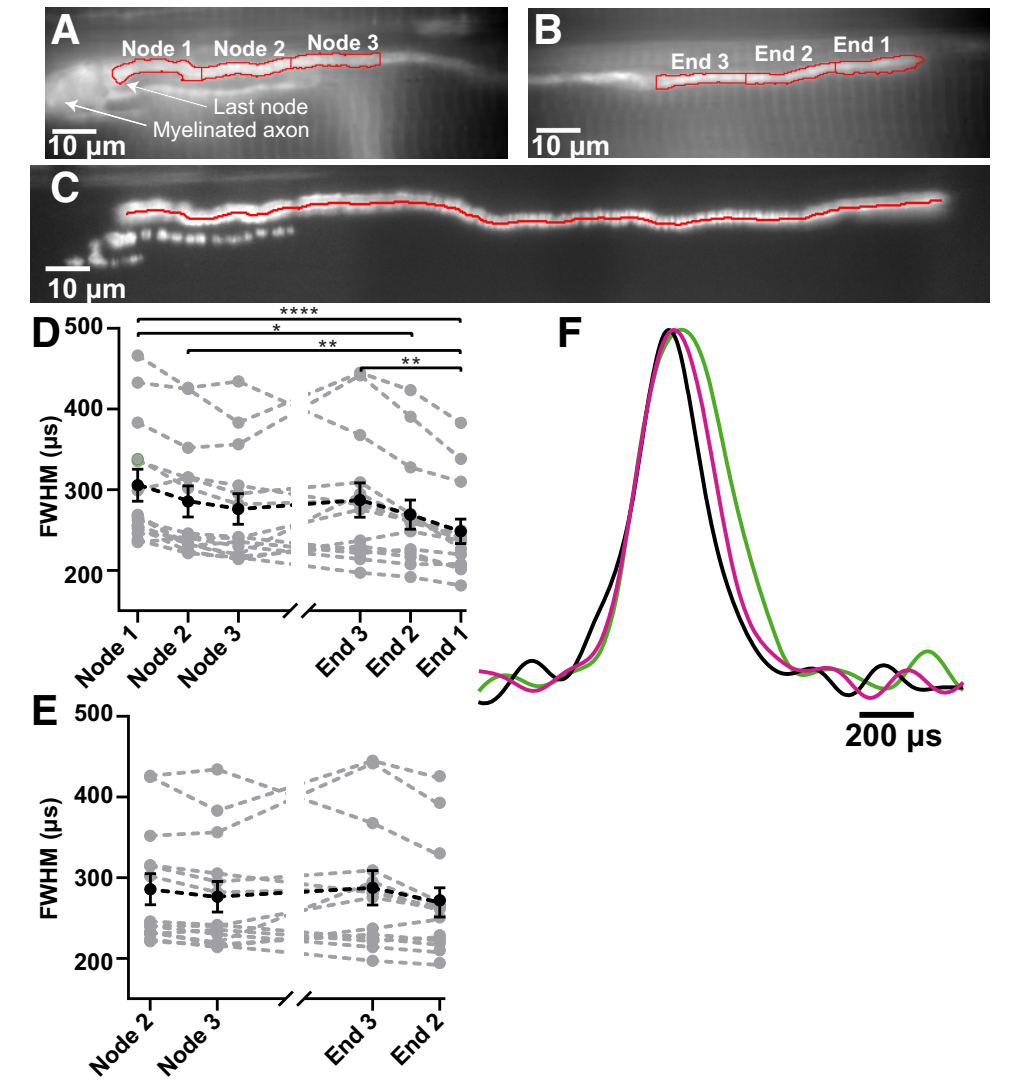

Figure 7. There are three distinct electrical regions along the frog motoneuron terminal. $A, A$ BeRST 1 dye image of nerve terminal near the last node of Ranvier. The myelinated axon and the last node of Ranvier are labeled in this image, along with the first, second, and third 20- $\mu$ m-long ROIs identified by red outlines (labeled Node 1, Node 2, Node 3) starting from the last node of Ranvier and moving into a long nerve terminal branch. $\boldsymbol{B}, \mathrm{A}$ BeRST 1 dye image of nerve terminal near the end of a long nerve terminal branch with the first, second, and third $20 \mu \mathrm{m}$ long ROls identified by red outlines (labeled End 1, End 2, and End 3), starting from the end of the nerve terminal. C, A BTX-labeled image of the full length of the long nerve terminal branch shown in $\boldsymbol{A}$ and $\boldsymbol{B}$, overlaid with the skeletonized version of this nerve terminal branch (red). D, Plot of imaged AP FWHM data derived from 14 long motor nerve terminal branches (gray symbols, one set of which was taken from $\boldsymbol{A}$ and $\boldsymbol{B}$ ) for each of $6 \mathrm{ROls} ; 3$ near the last node of Ranvier and 3 near the end of the nerve terminal branch (as shown in $\boldsymbol{A}$ and $\boldsymbol{B}$ ). The mean data ( \pm SEM) are shown using black symbols. Statistical significance between ROls along the terminal is determined by Friedman test with Dunn's multiple post hoc comparisons test. ${ }^{*} p<0.05$, ${ }^{* *} p<0.01,{ }^{* * * *} p<0.0001$. $E$, Plot of FWHM data from the four ROls in the middle region of long nerve terminal branches (gray symbols). Summary data are shown in black symbols. Within these middle regions (Node 2-End 2), there is no significant difference between any of these ROls. $p=0.0633$, Friedman test. $\boldsymbol{F}$, AP waveforms from Node 1 (green), End 3 (magenta), and End 1 (black) taken from a representative motor nerve terminal branch.

To further examine this hypothesis and to ensure that our results were not an artifact of our method of ROI selection for the data analysis, we reanalyzed the images by placing five ROIs, each consisting of 10-pixel diameter $(2.667 \mu \mathrm{m})$ circles spaced $15 \mu \mathrm{m}$ apart from their center along the nerve terminal just after the last node of Ranvier and near the end of the nerve terminal (Fig. 8A,B). Because these data are created with a different method of analysis than the $20 \mu \mathrm{m}$ ROIs, multiple-comparison corrections were performed separately for the $20 \mu \mathrm{m}$ ROI and small-circle ROI data. Consistent with our analysis of $20 \mu \mathrm{m}$ ROIs, these small-circle ROIs revealed significant differences near the last node of Ranvier where the axon enters the nerve terminal and near the end of the nerve terminal, but no significant differences along the long middle portion of the nerve terminal (Fig. 8C,D).

To test whether these changes in the AP waveform could be caused by passive propagation along the terminal, we created a passive model of the frog NMJ nerve terminal in NEURON and stimulated it with a recorded AP waveform from near the last node of Ranvier (Figs. 7F, 9A, 12A). Our model predicted that passive propagation would result in a broadening of the AP waveform as its amplitude decayed (Fig. 9). Furthermore, our model predicted that passive propagation would also cause broadening under a wide variety of different values for axon diameter, leak conductance, axial resistance, and membrane capacitance (data not shown). These model results of passive propagation were not consistent with our imaged AP waveform data.

Our imaged AP waveform data confirm the existence of three distinct electrical regions within the presynaptic nerve terminal. Furthermore, the lack of broadening due to decay in the AP waveform as it propagates $\sim 200 \mu \mathrm{m}$ from near the last node of Ranvier to near the end of the nerve terminal suggests that the AP is actively propagated throughout the presynaptic motor nerve terminal in frogs (consistent with predictions from early studies; Katz and Miledi, 1965, 1968; Braun and Schmidt, 1966).

\section{The propagation speed of the AP waveform does not change along the length of the terminal}

The propagation speed of the AP waveform along the length of the presynaptic motor nerve terminal of the frog NMJ has previously been experimentally estimated using extracellular recording techniques (Katz and Miledi, 1965; Braun and Schmidt, 1966; Nikol'kii et al., 2002). Furthermore, it had been suggested that the AP propagates at different speeds in different parts of the nerve terminal (Braun and Schmidt, 1966; Nikol'kii et al., 2002). However, these prior studies relied on reconstructing the electrical activity within the nerve terminal based on recordings from extracellular currents, which are prone to significant variation due to variances in the extracellular tissue surrounding the nerve terminal and the placement of the electrode with respect to the nerve terminal. To directly examine the propagation speed of the AP in the adult frog NMJ nerve terminal, we compared the time between the half-maximums of the rising edge of the AP waveforms from the small-circle ROI voltage imaging data from near the last node of Ranvier and at the tip of the nerve terminal branch (Fig. 8, CN1 and CE1). By combining these data with the distances recorded from the stitched BTX images of the total nerve terminal branch length, we were able to measure the propagation speed of APs $(0.6586 \pm 0.0700 \mathrm{~m} / \mathrm{s})$. We also compared the propagation speed between the first (CN1) and last (CN5) ROIs near the last node of Ranvier $(0.6006 \pm 0.0588 \mathrm{~m} / \mathrm{s})$ and the first (CE5) and last (CE1) ROIs near the end of the nerve terminal $(0.6584 \pm 0.0828 \mathrm{~m} / \mathrm{s})$ to determine whether the AP was propagating at different speeds near the last node of versus the end of the nerve terminal branches. There was no significant difference in AP propagation speed found between any of these three measurements, and the 
average between the three groups was $0.6392 \pm 0.0403 \mathrm{~m} / \mathrm{s}$. We repeated this analysis using our larger $20 \mu \mathrm{m}$ ROI data by assuming that the AP represents the center of the ROI (so the distance between the first and last ROIs from each image was assumed to be only $40 \mu \mathrm{m})$ and found almost identical results (Fig. 10). These results are consistent with the average speeds determined by extracellular recordings (Katz and Miledi, 1965; Braun and Schmidt, 1966; Nikol'kii et al., 2002), but do not agree with the extracellular data that had suggested that the AP propagates at different speeds in different parts of the motor nerve terminal (Braun and Schmidt, 1966; Nikol'kii et al., 2002). Furthermore, this propagation speed is similar to the AP propagation speed determined in Drosophila NMJs when imaging a genetically encoded voltage sensor (Ford and Davis, 2014), although the AP of the Drosophila NMJ is $\sim$ six times broader than the AP waveforms recorded at the frog NMJ.
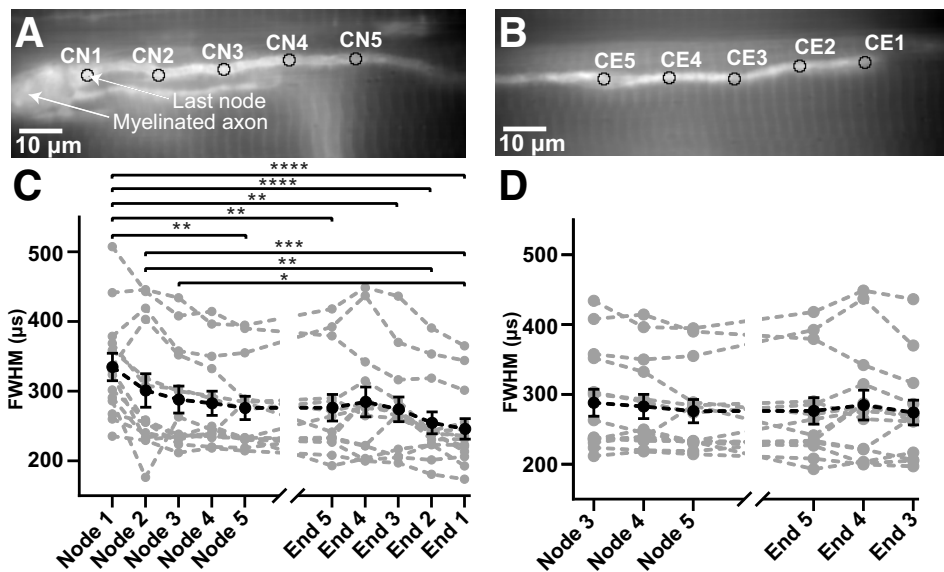

D

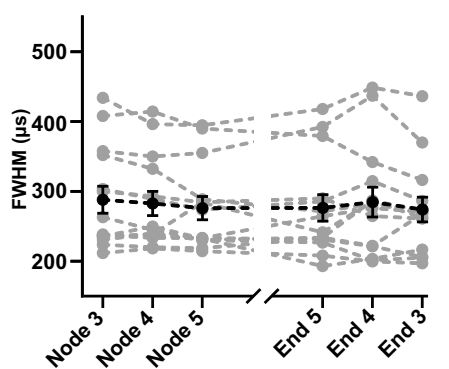

Figure 8. The use of small-circle ROls for image analysis confirms the presence of three distinct electrical regions within motor nerve terminal branches. A, A BeRST 1 dye image of a representative nerve terminal branch near the last node of Ranvier. The region of the motor nerve terminal near the last node of Ranvier is sampled with five circle R0ls placed $15 \mu \mathrm{m}$ apart, labeled Circle Node 1 (CN1) through CN5. Each circle is 10 pixels $(2.667 \mu \mathrm{m})$ in diameter. $\boldsymbol{B}, \mathrm{A}$ BeRST 1 dye image of a representative nerve terminal branch near the end of the nerve terminal with five circle ROls place $15 \mu \mathrm{m}$ apart, labeled Circle End 1 (CE1) through CE5. C, Plot of imaged AP FWHM data derived from 14 long motor nerve terminal branches (gray symbols, one of which was taken from $\boldsymbol{A}$ and $\boldsymbol{B}$ ) for each of the 10 ROls identified in $\boldsymbol{A}$ and $\boldsymbol{B}$. The mean data ( \pm SEM) are shown using black symbols. Statistical significance between Rols along the terminal is determined by Friedman test with Dunn's multiple post hoc comparisons test. ${ }^{*} p<0.05,{ }^{* *} p<0.01,{ }^{* * *} p<0.001,{ }^{* * * *} p<0.0001$. D, Plot of data from six Rols in the middle region of long nerve terminal branches (gray symbols), with summary data (black symbols). Within this middle region (CN3(E3), there is no significant difference between any of these R0ls. $p=0.2161$, Friedman test.

\section{Motor nerve terminal width does not} correlate with AP duration or propagation speed within the motor nerve terminal

Although nerve terminal diameter would be predicted to play a role in determining the shape and propagation speed of the AP waveform, research in other neuron types has reported that the diameter of the presynaptic nerve terminal has a negligible effect on the shape and propagation of AP waveforms compared with the effect of ion channel expression (Rowan et al., 2016). To investigate the impact of nerve terminal geometry on the AP waveform and to test whether changes in nerve terminal geometry could be responsible for the observed changes in the frog NMJ AP waveform, we measured the area of the imaged nerve terminal region contained in all three of the $20 \mu \mathrm{m}$ ROIs near the last node of Ranvier, as well as near the end of the nerve terminal. By dividing this area by the length between the two first and last ROIs, we calculated the average width of the axon near the last node of Ranvier and at the end of the nerve terminal. We then calculated the correlation between axon width, the $20 \mu \mathrm{m}$ ROI AP FWHM, and the $20 \mu \mathrm{m}$ ROI propagation speeds in a combined dataset of the values near the end of the terminal and near the last node of Ranvier (where the AP FWHMs of the three $20 \mu \mathrm{m}$ ROIs near the last node of Ranvier are averaged together for each terminal, and the same is done for the three $20 \mu \mathrm{m}$ ROIs near the end of each terminal). We note that our imaging procedure was limited to measuring the width of the nerve terminal, so our analysis of the terminal geometry was based on a two-dimensional image assuming a symmetrical three-dimensional geometry. Using this approach, we found no correlation between terminal width and either AP FWHM or AP propagation speed but did find a correlation between AP FWHM and AP propagation speed (Fig. 11A,C,E). We then repeated this analysis using propagation speed and AP FWHM values from our small-circle ROIs and found similar results (Fig. 11B,D,F).
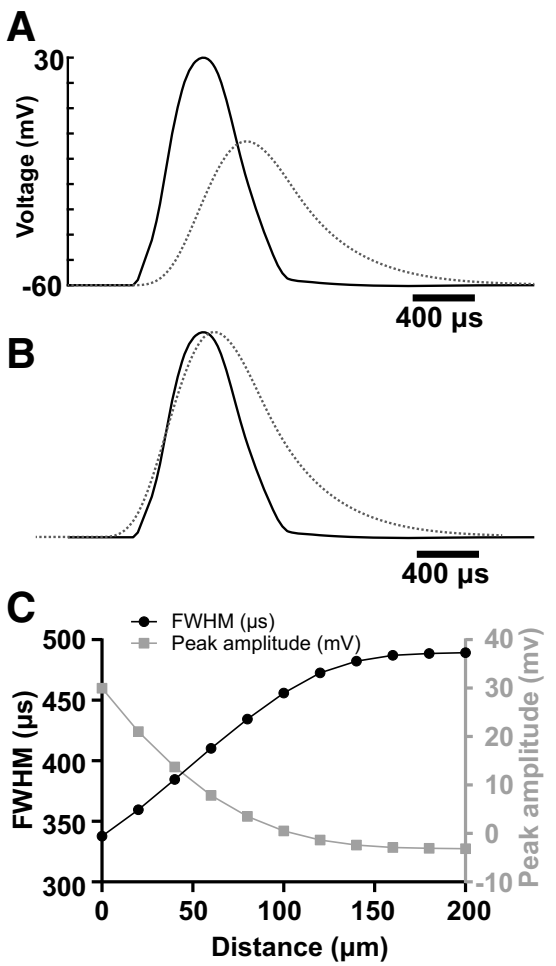

Figure 9. NEURON simulation predictions for changes to the AP waveform caused by passive propagation over a $200 \mu \mathrm{m}$ long nerve terminal. $A$, waveform input based on recorded AP waveforms from near the last node of Ranvier (black), and the predicted change to the AP waveform caused by passive decay to the end of the long nerve terminal branch (gray dotted line). $\boldsymbol{B}$, The two waveforms from $\boldsymbol{A}$ normalized and aligned to the half-maximum of their rising edge, showing a predicted $44 \%$ increase in the FWHM between the AP near the last node of Ranvier and near the end of the terminal. $\boldsymbol{C}$, The predicted FWHM (black) and amplitude (gray) of the AP waveform (measured every $20 \mu \mathrm{m}$ ) as it passively propagates along a $200 \mu \mathrm{m}$ long nerve terminal. 


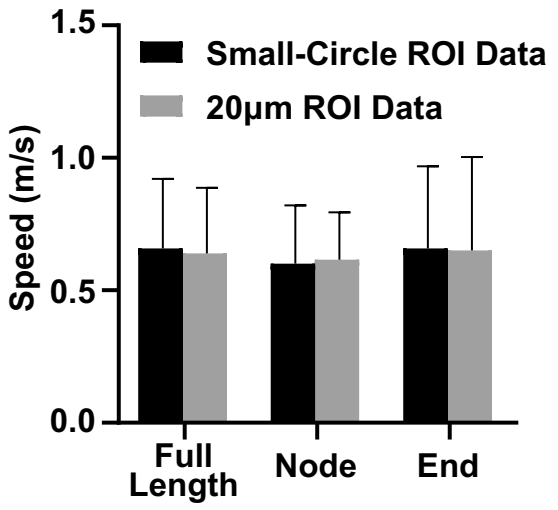

Figure 10. Comparison of the AP propagation speeds near the last node of Ranvier, near the end of the terminal, and along the length of the entire terminal using both $20 \mu \mathrm{m} \mathrm{ROI}$ and small-circle ROl data (mean + S.D.). There is no significant difference in AP propagation speed in different regions of the terminal $(n=14 ; p=0.7515$ for $20 \mu \mathrm{m}$ ROls, $p=0.3189$ for small-circle Rols; Friedman test), and there is no significant difference between the $20 \mu \mathrm{m}$ and small-circle ROI measurements in any region (full length: $p=0.5416$, node: $p=0.2166$, end: $p=0.5830$; Wilcoxon matched-pairs signed rank test).
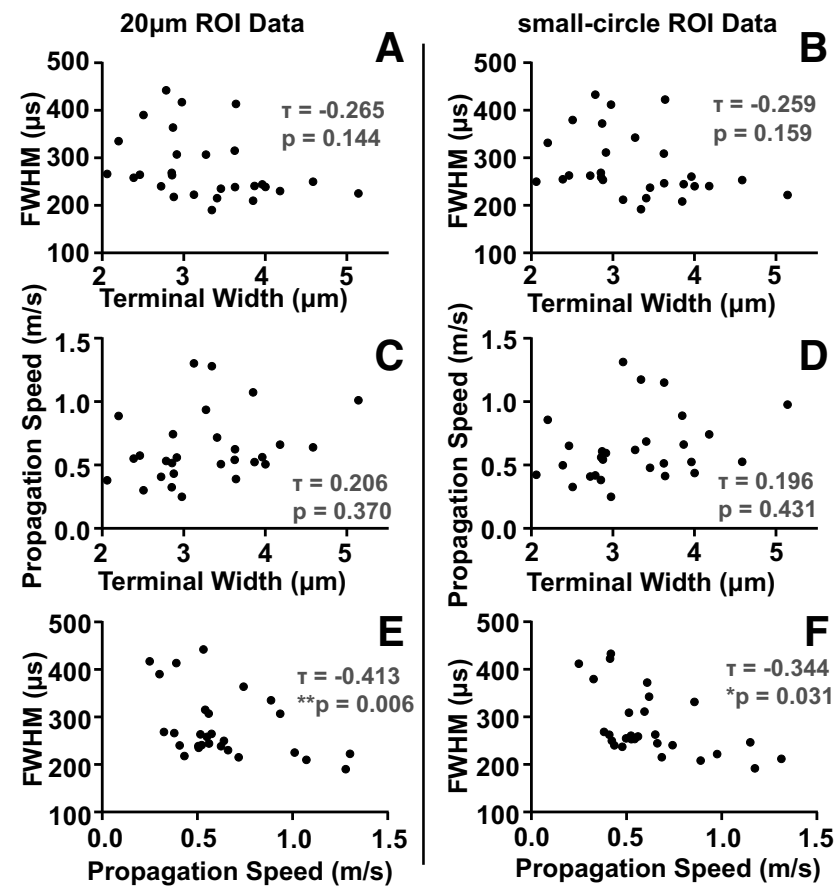

Figure 11. Combined data from near the end of the terminal and near the last node of Ranvier $(n=28)$, using both the $20 \mu \mathrm{m}$ ROls (left) and small circle ROls (right), showing that measured motor nerve terminal width does not correlate with either AP duration (FWHM) or propagation speed, but propagation speed correlates with AP FWHM. $\boldsymbol{A}, \boldsymbol{B}$, Lack of correlation between the measured motor nerve terminal widths and AP durations (FWHM). C, D, Lack of correlation between the measured motor nerve terminal widths and the propagation speeds of the APs. $\boldsymbol{E}, \boldsymbol{F}$, Significant correlation between the AP duration (FWHM) and the AP propagation speed. All correlations are calculated with Kendall's $\tau$ coefficient, and $p$ values were adjusted using Bonferroni's correction.

\section{Discussion}

The shape of the AP waveform at the motor nerve terminal impacts the behavior of presynaptic VGCCs, and thus is an important modulator of neurotransmitter release at the presynaptic nerve terminal. Although the adult frog NMJ has long been a model synapse for the study of synaptic transmission, its AP
A

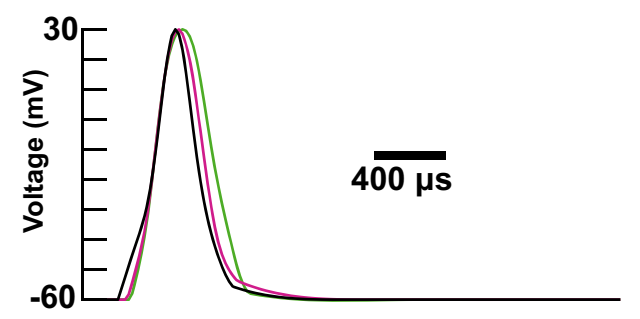

B
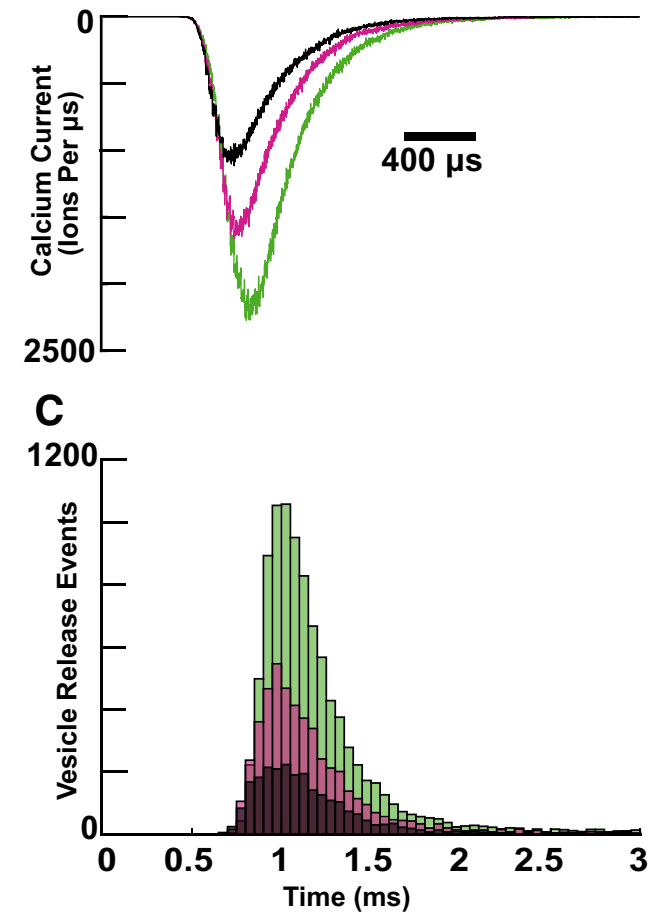

Figure 12. MCell predictions for calcium entry and transmitter release induced by AP waveforms measured from three distinct electrical regions of the nerve terminal. $A$, AP waveforms used for computational simulations based on the recorded AP waveforms from near the last node of Ranvier (green), the middle region of the nerve terminal (magenta), and near the end of the nerve terminal (black). B, Predicted calcium current induced by each AP near the last node of Ranvier (green), the middle region of the nerve terminal (magenta), and the end of the nerve terminal (black) based on a MCell box simulation of 10,000 VGCCs. C, Histogram of the time course of vesicle release events predicted from 4800 runs of the frog active zone MCell model in response to calcium-entry triggered vesicle fusion induced by the APs measured from near the last node of Ranvier (green), the region of the nerve terminal (magenta), and the end of the nerve terminal (black).

waveform has never been directly recorded. The lack of information regarding this AP waveform has hindered the ability to investigate the voltage-driven control of synaptic transmission, and also impacts computational simulations which depend on AP waveform-driven ion channel gating and ion flux (Dittrich et al., 2013; Ma et al., 2014; Homan et al., 2018; Laghaei et al., 2018). Furthermore, there has been debate as to whether APs within the long linear presynaptic terminals of the adult frog NMJ are actively or passively propagated. Therefore, to examine the duration and propagation of the presynaptic AP at the frog NMJ, we used a voltage-sensitive dye (BeRST 1) and showed that the AP waveform is extremely brief and is actively propagated throughout the nerve terminal.

For the data reported here, we selected nerve terminals for our imaging procedure that contained long $(>120 \mu \mathrm{m})$, linear 
nerve terminal branches. Thus, it is possible that short nerve terminals or terminals with significant branching may display different changes in their AP waveform duration.

\section{Very brief AP waveforms may aid in maintaining strength and reliability at the NMJ}

Voltage imaging of the presynaptic terminal of the frog NMJ revealed that the average AP waveform had a FWHM of only $273.9 \pm$ $10.7 \mu \mathrm{s}$. This is much briefer than expected based on electrophysiological recordings from both adult frog motoneuron cell bodies in the spinal cord (Dambach and Erulkar, 1973; Erulkar and Soller, 1980; Ovsepian and Vesselkin, 2006), and cultured frog embryonic motoneuron synaptic varicosities onto muscle cells (Yazejian et al., 1997; Pattillo et al., 2001). This leads us to wonder what advantages for adult NMJ function might be provided by a very narrow $\mathrm{AP}$ at these motor nerve terminals?

This question has been addressed in detail within the central nervous system at the calyx of Held synapse; a very large synapse in the mammalian auditory brainstem that functions to differentiate between temporal activation of cochlear hair cells in each ear to aid in sound localization (Tsuchitani, 1997). The AP at the mammalian calyx of Held has been shown to become briefer in duration over development (560 $\mu$ s FWHM before hearing onset and $190 \mu$ s after hearing onset), but has been shown not to change in amplitude over this same time period (Taschenberger and von Gersdorff, 2000). This AP duration change is thought to be caused by changes in presynaptic voltage-gated sodium channels (faster inactivation and recovery) and potassium channels (higher density and faster activation kinetics; Elezgarai et al., 2003; Leao et al., 2005; Nakamura and Takahashi, 2007). The very short duration of mature APs at the calyx of Held reduces the activation of presynaptic VGCCs, decreasing the probability of release from each of the $\sim 700 \mathrm{AZs}$ (Borst and Sakmann, 1998; Taschenberger et al., 2002; Yang and Wang, 2006). These and other developmental refinements at the calyx of Held lead to fast and dependable communication at this relay synapse. The NMJ is also a strong and reliable relay synapse that is expected to release enough transmitter with each AP to trigger postsynaptic muscle contraction (Wood and Slater, 2001). The NMJ accomplishes this strength and reliability by assembling hundreds of presynaptic AZs that each contains 2040 single vesicle release sites (docked vesicles and their associated VGCCs). In contrast to the calyx of Held synapse, the frog NMJ does not show frequency-dependent depression, and in fact typically displays facilitation during pairs or short trains of APs (Laghaei et al., 2018). This is likely due in part to the very low probability for release at each of the thousands of single vesicle release sites contained within hundreds of AZs (Tarr et al., 2013). In this way, a very brief presynaptic AP can ensure that very few presynaptic VGCCs open (Luo et al., 2011, 2015), sparing most release sites from participating in AP-evoked release. This mechanism may aid in preserving transmitter release-ready capability during subsequent APs, even at high frequency.
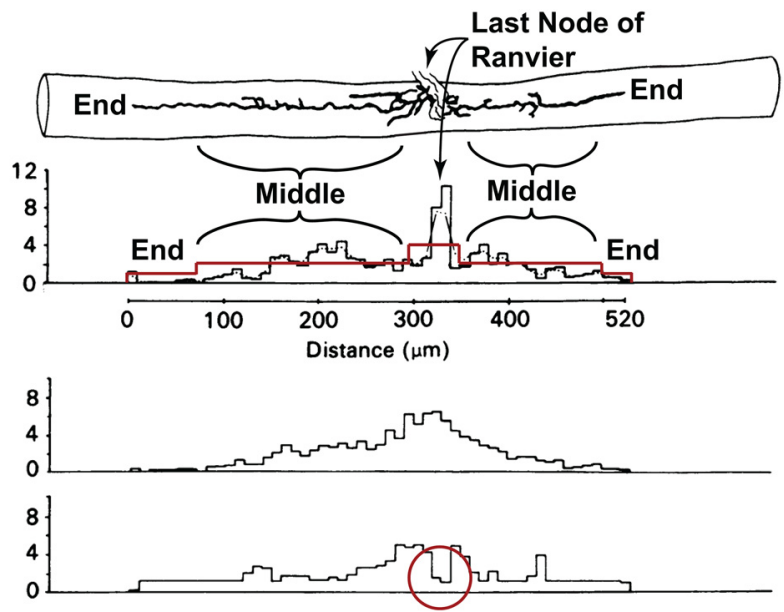

f a single adult frog NMJ $(\boldsymbol{A})$ and the associated histograms of the measured probability of release along the length of this nerve terminal. In this figure, adapted from D'Alonzo and Grinnell (1985), the probability of release per unit nerve length $(\boldsymbol{B})$ is greatest near the last node of Ranvier, relatively constant through the middle region of the terminal. Red line shows changes in transmitter release predicted by our measured nerve terminal length. Red circle identifies the last node of Ranvier where measured nerve terminal length is

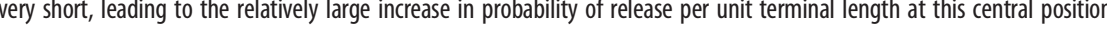
transmitter release probability along the length of this sample NMJ. Therefore, we hypothesize that these changes in transmitter release could be caused by the changes in the AP waveform we measured along the length of similar frog NMJs.

\section{Changes in the duration of the AP waveform could explain proximal-distal changes in neurotransmitter release}

To computationally predict how proximal-distal changes in the AP waveform could alter calcium triggered transmitter release, we input our experimentally-imaged AP waveforms from the three regions of the presynaptic nerve terminal of the frog $\mathrm{NMJ}$ (Fig. $7 F$ ) into an MCell simulation of 10,000 VGCCs (Fig. 12A; Materials and Methods). This simulation predicted that the probability of VGCCs opening during a single AP were 0.203 , 0.307, and 0.436 for APs from near the end, the middle, and near the last node, respectively, and also predicted a $48 \%$ increase in total calcium flux between the end and middle AP waveform, and a $119 \%$ increase in calcium flux between the nerve terminal end and AP waveform near the last node (Fig. 12B). Next, we used these APs in our previously validated MCell model of a frog AZ (Materials and Methods; Dittrich et al., 2013; Ma et al., 2014; Homan et al., 2018; Laghaei et al., 2018). The frog AZ model predicted large differences in the magnitude of transmitter release caused by the APs recorded from different regions of the motor nerve terminal, with the AP waveform from near the last node of Ranvier triggering approximately double the transmitter release as comparted to the AP waveform recorded near the middle, and approximately four times the transmitter release compared with the AP waveform recorded near the end of the nerve terminal (Fig. 12C). These computational results show that small changes in the width of the AP waveform can result in large changes in presynaptic calcium entry and transmitter release (Fig. 12B,C). Furthermore, these results are consistent with the proximal-distal gradient in the magnitude of transmitter release along the frog motor nerve terminal reported by D'Alonzo and Grinnell (1985). Therefore, we hypothesize that changes in the AP waveform along the length of the adult frog motor nerve terminal may be responsible for the proximal-distal decrease in neurotransmitter release found at the presynaptic nerve terminal of the frog NMJ (Fig. 13). 


\section{Relationship between axon width, the duration of the AP waveform, and AP propagation speed}

We measured the propagation speed along the length of the adult frog motor nerve terminals and determined the average to be $\sim 0.6 \mathrm{~m} / \mathrm{s}$. This value is relatively slow when compared with the range of propagation speeds previously measured in mammalian peripheral axons, (even when accounting for temperature differences, for which a Q10 of 1.5 has been reported for AP conduction velocities at the squid giant synapse; Rosenthal and Bezanilla, 2000), which can range from $\sim 120 \mathrm{~m} / \mathrm{s}$ for the fastest large diameter $(\sim 20 \mu \mathrm{m})$ myelinated axons, to $\sim 0.3 \mathrm{~m} / \mathrm{s}$ for small diameter $(\sim 0.1 \mu \mathrm{m})$ unmyelinated axons (Swadlow and Waxman, 2012). Theoretically, the diameter of a nerve terminal should impact the AP waveform shape and propagation speed along a long linear nerve terminal. However, by comparing the width of the nerve terminal with both the FWHM of the AP waveform and the AP propagation speed we found no significant correlation. We hypothesize that this is because the distribution of ion channels plays a much larger role in determining the shape of the AP waveform such that modest changes in nerve terminal geometry have a negligible impact. Neurons are known to use a large variety of voltage-gated sodium and potassium channels that can lead to different AP shapes (Bean, 2007). In particular, fast-spiking neurons that employ very brief APs express higher densities of specific types of voltage-gated potassium channels (Rudy and McBain, 2001). In fact, even within the same neuron, APs can differ significantly when comparing the soma with the nerve terminal because of a non-uniform distribution of voltagegated sodium and potassium channels (Geiger and Jonas, 2000). It is thought that the presynaptic terminal of the frog NMJ contains voltage-gated sodium channels (although the subtype is unknown, they are expected to be similar to the TTX-sensitive channels reported in mammalian axons; Caldwell et al., 2000), and BK channels have been localized selectively to AZs within frog NMJs (Robitaille et al., 1993a,b). The types of voltage-gated potassium channels are unknown, but we hypothesize that the presynaptic terminal of the frog NMJ might contain Kv3 subtypes based on reports in mammalian motor nerve terminals (Brooke et al., 2004; Zemel et al., 2018). Despite decades of study, with the exception of AZ-localized BK channels (Robitaille et al., 1993a,b), little is known of the expression of sodium and potassium channels within the adult frog motor nerve terminal. Further studies aimed at elucidating the density and distribution of these channels within the frog NMJ will be required to determine the mechanisms responsible for changes in the AP waveform.

\section{References}

Arganda-Carreras I, Fernández-González R, Muñoz-Barrutia A, Ortiz-DeSolorzano C (2010) 3D reconstruction of histological sections: application to mammary gland tissue. Microsc Res Tech 73:1019-1029.

Bean BP (2007) The action potential in mammalian central neurons. Nat Rev Neurosci 8:451-465.

Beier HT, Roth CC, Bixler JN, Sedelnikova AV, Ibey BL (2019) Visualization of dynamic sub-microsecond changes in membrane potential. Biophys J 116:120-126.

Bennett M, Jones P, Lavidis N (1986a) Transmitter secretion varies between visualized release sites at amphibian neuromuscular junctions. Neurosci Lett 65:311-315.

Bennett MR, Jones P, Lavidis NA (1986b) The probability of quantal secretion along visualized terminal branches at amphibian (Bufo marinus) neuromuscular synapses. J Physiol 379:257-274.

Bennett MR, Lavidis NA, Lavidis-Armson F (1989) The probability of quantal secretion at release sites of different length in toad (Bufo marinus) muscle. J Physiol 418:235-249.
Borst JG, Sakmann B (1998) Calcium current during a single action potential in a large presynaptic terminal of the rat brainstem. J Physiol 506: 143-157.

Borst JG, Sakmann B (1999) Effect of changes in action potential shape on calcium currents and transmitter release in a calyx-type synapse of the rat auditory brainstem. Philos Trans R Soc Lond B Biol Sci 354:347-355.

Braun M, Schmidt RF (1966) Potential changes recorded from the frog motor nerve terminal during its activation. Pflugers Arch Gesamte Physiol Menschen Tiere 287:56-80.

Brooke RE, Moores TS, Morris NP, Parson SH, Deuchars J (2004) Kv3 voltage-gated potassium channels regulate neurotransmitter release from mouse motor nerve terminals. Eur J Neurosci 20:3313-3321.

Caldwell JH, Schaller KL, Lasher RS, Peles E, Levinson SR (2000) Sodium channel $\mathrm{Na}(\mathrm{v}) 1.6$ is localized at nodes of ranvier, dendrites, and synapses. Proc Natl Acad Sci U S A 97:5616-5620.

Cohen MW, Jones OT, Angelides KJ (1991) Distribution of $\mathrm{Ca}^{2+}$ channels on frog motor nerve terminals revealed by fluorescent omega-conotoxin. J Neurosci 11:1032-1039.

D'Alonzo AJ, Grinnell AD (1985) Profiles of evoked release along the length of frog motor nerve terminals. J Physiol 359:235-258.

Dambach GE, Erulkar SD (1973) The action of calcium at spinal neurones of the frog. J Physiol 228:799-817.

Dittrich M, Pattillo JM, King JD, Cho S, Stiles JR, Meriney SD (2013) An excess-calcium-binding-site model predicts neurotransmitter release at the neuromuscular junction. Biophys J 104:2751-2763.

Dodge FA Jr, Rahamimoff R (1967) Co-operative action a calcium ions in transmitter release at the neuromuscular junction. J Physiol 193:419-432.

Elezgarai I, Diez J, Puente N, Azkue JJ, Benitez R, Bilbao A, Knopfel T, Donate-Oliver F, Grandes P (2003) Subcellular localization of the voltage-dependent potassium channel $\mathrm{Kv} 3.1 \mathrm{~b}$ in postnatal and adult rat medial nucleus of the trapezoid body. Neuroscience 118:889-898.

Erulkar SD, Soller RW (1980) Interactions among lumbar motoneurons on opposite sides of the frog spinal cord: morphological and electrophysiological studies. J Comp Neur 192:473-488.

Ford KJ, Davis GW (2014) Archaerhodopsin voltage imaging: synaptic calcium and BK channels stabilize action potential repolarization at the Drosophila neuromuscular junction. J Neurosci 34:14517-14525.

Geiger JR, Jonas P (2000) Dynamic control of presynaptic $\mathrm{Ca}^{2+}$ inflow by fast-inactivating $\mathrm{K}(+)$ channels in hippocampal mossy fiber boutons. Neuron 28:927-939.

Grinnell AD, Herrera AA (1980) Physiological regulation of synaptic effectiveness at frog neuromuscular junctions. J Physiol 307:301-317.

Heredia DJ, Schubert D, Maligireddy S, Hennig GW, Gould TW (2016) A novel striated muscle-specific myosin-blocking drug for the study of neuromuscular physiology. Front Cell Neurosci 10:276.

Heuser JE, Reese TS (1981) Structural changes after transmitter release at the frog neuromuscular junction. J Cell Biol 88:564-580.

Hines ML, Carnevale NT (1997) The NEURON simulation environment. Neural Comput 9:1179-1209.

Hines ML, Carnevale NT (2001) NEURON: a tool for neuroscientists. Neuroscientist 7:123-135.

Homan AE, Laghaei R, Dittrich M, Meriney SD (2018) Impact of spatiotemporal calcium dynamics within presynaptic active zones on synaptic delay at the frog neuromuscular junction. J Neurophysiol 119:688-699.

Hoppa MB, Gouzer G, Armbruster M, Ryan TA (2014) Control and plasticity of the presynaptic action potential waveform at small CNS nerve terminals. Neuron 84:778-789.

Huang YL, Walker AS, Miller EW (2015) A Photostable Silicon Rhodamine Platform for Optical Voltage Sensing. J Am Chem Soc 137:10767-10776.

Katz B, Miledi R (1965) Propagation of electric activity in motor nerve terminals. Proc R Soc Lond B Biol Sci 161:453-482.

Katz B, Miledi R (1968) The effect of local blockage of motor nerve terminals. J Physiol 199:729-741.

Kerr RA, Bartol TM, Kaminsky B, Dittrich M, Chang JC, Baden SB, Sejnowski TJ, Stiles JR (2008) Fast Monte Carlo simulation methods for biological reaction-diffusion systems in solution and on surfaces. SIAM J Sci Comput 30:3126.

Laghaei R, Ma J, Tarr TB, Homan AE, Kelly L, Tilvawala MS, Vuocolo BS, Rajasekaran HP, Meriney SD, Dittrich M (2018) Transmitter release site organization can predict synaptic function at the neuromuscular junction. J Neurophysiol 119:1340-1355.

Leao RM, Kushmerick C, Pinaud R, Renden R, Li GL, Taschenberger H, Spirou G, Levinson SR, von Gersdorff H (2005) Presynaptic Na+ 
channels: locus, development, and recovery from inactivation at a high-fidelity synapse. J Neurosci 25:3724-3738.

Lindgren CA, Moore JW (1989) Identification of ionic currents at presynaptic nerve endings of the lizard. J Physiol 414:201-222.

Luo F, Dittrich M, Stiles JR, Meriney SD (2011) Single-pixel optical fluctuation analysis of calcium channel function in active zones of motor nerve terminals. J Neurosci 31:11268-11281.

Luo F, Dittrich M, Cho S, Stiles JR, Meriney SD (2015) Transmitter release is evoked with low probability predominately by calcium flux through single channel openings at the frog neuromuscular junction. J Neurophysiol 113:2480-2489.

Ma J, Kelly L, Ingram J, Price TJ, Meriney SD, Dittrich M (2014) New insights into short-term synaptic facilitation at the frog neuromuscular junction. J Neurophysiol 113:71-87.

Mallart A (1984) Presynaptic currents in frog motor endings. Pflugers Arch 400:8-13.

Maric D, Maric I, Smith SV, Serafini R, Hu Q, Barker JL (1998) Potentiometric study of resting potential, contributing $\mathrm{K}+$ channels and the onset of $\mathrm{Na}+$ channel excitability in embryonic rat cortical cells. Eur J Neurosci 10:2532-2546.

Meriney SD, Dittrich M (2013) Organization and function of transmitter release sites at the neuromuscular junction. J Physiol 591:3159-3165.

Meunier FM (1984) Relationship between presynaptic membrane potential and acetylcholine release in synaptosomes from Torpedo electric organ. J Physiol 354:121-137.

Miralles F, Solsona C (1996) Activity-dependent modulation of the presynaptic potassium current in the frog neuromuscular junction. J Physiol 495: $717-732$.

Nakamura Y, Takahashi T (2007) Developmental changes in potassium currents at the rat calyx of Held presynaptic terminal. J Physiol 581:1101-1112.

Nikol'kii EE, Bukharaeva EA, Samigullin DV, Gainulo RK (2002) Characteristics of the time course of evoked secretion of transmitter quanta in different parts of the motor nerve ending in the frog. Neurosci Behav Physiol 32:265-274.

Otsu N (1979) A Threshold Selection Method from Gray-Level Histograms. IEEE Trans Syst Man Cybern 9:62-66.

Ovsepian SV, Vesselkin NP (2006) Serotonergic modulation of synaptic transmission and action potential firing in frog motoneurons. Brain Res 1102:71-77.

Pattillo JM, Artim DE, Simples JE Jr, Meriney SD (1999) Variations in onset of action potential broadening: effects on calcium current studied in chick ciliary ganglion neurones. J Physiol 514:719-728.

Pattillo JM, Yazejian B, DiGregorio DA, Vergara JL, Grinnell AD, Meriney SD (2001) Contribution of presynaptic calcium-activated potassium currents to transmitter release regulation in cultured Xenopus nerve-muscle synapses. Neuroscience 102:229-240.

Pawson PA, Grinnell AD, Wolowske B (1998) Quantitative freeze-fracture analysis of the frog neuromuscular junction synapse-II. Proximal-distal measurements. J Neurocytol 27:379-391.

Podleski T, Changeux JP (1969) Effects associated with permeability changes caused by gramicidin A in electroplax membrane. Nature 221:541-545.

Popovic MA, Foust AJ, McCormick DA, Zecevic D (2011) The spatio-temporal characteristics of action potential initiation in layer 5 pyramidal neurons: a voltage imaging study. J Physiol 589:4167-4187.

Preibisch S, Saalfeld S, Tomancak P (2009) Globally optimal stitching of tiled 3D microscopic image acquisitions. Bioinformatics 25:1463-1465.

Pumplin DW, Reese TS, Llinas R (1981) Are the presynaptic membrane particles the calcium channels? Proc Natl Acad Sci U S A 78:7210-7213.

Robitaille R, Tremblay JP (1987) Non-uniform release at the frog neuromuscular junction: evidence of morphological and physiological plasticity. Brain Res 434:95-116.

Robitaille R, Adler EM, Charlton MP (1990) Strategic location of calcium channels at transmitter release sites of frog neuromuscular synapses. Neuron 5:773-779.
Robitaille R, Adler EM, Charlton MP (1993a) Calcium channels and calcium-gated potassium channels at the frog neuromuscular junction. J Physiol Paris 87:15-24.

Robitaille R, Garcia ML, Kaczorowski GJ, Charlton MP (1993b) Functional colocalization of calcium and calcium-gated potassium channels in control of transmitter release. Neuron 11:645-655.

Rosenthal JJ, Bezanilla F (2000) Seasonal variation in conduction velocity of action potentials in squid giant axons. Biol Bull 199:135-143.

Rowan MJ, Tranquil E, Christie JM (2014) Distinct Kv channel subtypes contribute to differences in spike signaling properties in the axon initial segment and presynaptic boutons of cerebellar interneurons. J Neurosci 34:6611-6623.

Rowan MJ, DelCanto G, Yu JJ, Kamasawa N, Christie JM (2016) Synapselevel determination of action potential duration by $\mathrm{K}(+)$ channel clustering in axons. Neuron 91:370-383.

Rowan MJM, Christie JM (2017) Rapid state-dependent alteration in Kv3 channel availability drives flexible synaptic signaling dependent on somatic subthreshold depolarization. Cell Rep 18:2018-2029.

Rudy B, McBain CJ (2001) Kv3 channels: voltage-gated K+ channels designed for high-frequency repetitive firing. Trends Neurosci 24:517526.

Sabatini BL, Regehr WG (1997) Control of neurotransmitter release by presynaptic waveform at the granule cell to Purkinje cell synapse. J Neurosci 17:3425-3435.

Swadlow HA, Waxman SG (2012) Axonal conduction delays. Scholarpedia 7:1451.

Tarr TB, Malick W, Liang M, Valdomir G, Frasso M, Lacomis D, Reddel SW, Garcia-Ocano A, Wipf P, Meriney SD (2013) Evaluation of a novel calcium channel agonist for therapeutic potential in Lambert-Eaton myasthenic syndrome. J Neurosci 33:10559-10567.

Taschenberger H, von Gersdorff H (2000) Fine-tuning an auditory synapse for speed and fidelity: developmental changes in presynaptic waveform, EPSC kinetics, and synaptic plasticity. J Neurosci 20:9162-9173.

Taschenberger H, Leao RM, Rowland KC, Spirou GA, von Gersdorff $\mathrm{H}$ (2002) Optimizing synaptic architecture and efficiency for high-frequency transmission. Neuron 36:1127-1143.

Tremblay JP, Robitaille R, Grenon G (1984) Distribution of spontaneous release along the frog neuromuscular junction. Neurosci Lett 51:247252.

Tseng Q, Wang I, Duchemin-Pelletier E, Azioune A, Carpi N, Gao J, Filhol O, Piel M, Théry M, Balland M (2011) A new micropatterning method of soft substrates reveals that different tumorigenic signals can promote or reduce cell contraction levels. Lab Chip 11:2231-2240.

Tseng Q, Duchemin-Pelletier E, Deshiere A, Balland M, Guillou H, Filhol O, Théry M (2012) Spatial organization of the extracellular matrix regulates cell-cell junction positioning. Proc Natl Acad Sci U S A 109: 1506-1511.

Tsuchitani C (1997) Input from the medial nucleus of trapezoid body to an interaural level detector. Hear Res 105:211-224.

Wood SJ, Slater CR (2001) Safety factor at the neuromuscular junction. Prog Neurobiol 64:393-429.

Yang YM, Wang LY (2006) Amplitude and kinetics of action potentialevoked $\mathrm{Ca}^{2+}$ current and its efficacy in triggering transmitter release at the developing calyx of Held synapse. J Neurosci 26:5698-5708.

Yazejian B, DiGregorio DA, Vergara JL, Poage RE, Meriney SD, Grinnell AD (1997) Direct measurements of presynaptic calcium and calcium-activated potassium currents regulating neurotransmitter release at cultured Xenopus nerve-muscle synapses. J Neurosci 17:2990-3001.

Zefirov AL, Khalilov IA (1985) [Characteristics of electrical activity in different sections of nerve endings in the frog]. Biull Eksp Biol Med 99: $7-10$.

Zemel BM, Ritter DM, Covarrubias M, Muqeem T (2018) A-type KV channels in dorsal root ganglion neurons: diversity, function, and dysfunction. Front Mol Neurosci 11:253. 